\title{
SOLIDS AND LIQUIDS
}

\section{Microstructure of Bidisperse Ferrofluids in a Thin Layer ${ }^{1}$}

\author{
E. S. Minina ${ }^{a}$, A. B. Muratova ${ }^{a}$, J. J. Cerdá ${ }^{\prime}$, and S. S. Kantorovich ${ }^{a, b, c}$ \\ ${ }^{a}$ Department of Mathematical Physics, Ural Federal University, ul. Lenina 51, Yekaterinburg, 620000 Russia \\ ${ }^{b}$ Sapienza University of Rome, 00185, Piazza A. de Moro, Rome, Italy \\ ${ }^{c}$ Computational Physics Department, University of Vienna, Sensengasse 8, 1090 Wien, Austria \\ ${ }^{d}$ Institute for Cross-Disciplinary Physics and Complex Systems, Campus University de les Illes Balears, \\ 07122 Palma de Mallorca, Spain \\ e-mail:alla2307@gmail.com; sue.kantorovich@usu.ru
}

Received July 6, 2012

\begin{abstract}
In this work we present a characterization of the bidisperse ferrofluid microstructures that appear in thin layers of ferrofluid. These layers have been studied by a combination of Langevin dynamics simulations and density functional theory. Our results allow us to compare the microstructures that exist in quasi-twodimensional ferrofluid nanolayers with the microstructures found in three-dimensional bidisperse ferrofluids. Furthermore, our results allow us to explain the influence of the geometry of the sample on the topology and size-distribution of the observed aggregates of magnetic nanoparticles.
\end{abstract}

DOI: $10.1134 / \mathrm{S} 1063776113020027$

\section{INTRODUCTION}

Dipolar magnetic fluids (also known as ferrofluids or ferrocolloids) are colloidal suspensions of ferromagnetic single-domain nanoparticles in non-magnetic carriers [1]. Typical sizes of the particles are about $10-20 \mathrm{~nm}$. As a rule, magnetic fluids are stabilized by adsorbed layers (sterically stabilized magnetic fluids) or electrical double layers (ion-stabilized magnetic fluids). Those layers prevent particle agglomeration and sedimentation. Due to the magnetic nature of the particles, external magnetic fields induce reorientations of the particles which lead to changes in the properties of the magnetic fluids. The unique combination of fluidity and ability to interact with magnetic fields are the pillars of the wide range of applications of ferrocolloids that span from engineering to medicine [2-5].

Even in the absence of an external magnetic field, ferrofluids have quite a complex microstructure. In the thermodynamic equilibrium magnetic particles tend to form several types of structures whose shape depends on the system geometry, the magnetic interactions, and the degree of polydispersity. From the experimental point of view, the study of bulky ferrofluid microstructures is a highly nontrivial problem mainly due to the fact that ferroparticle particles have extremely small sizes and the liquid carriers are optically opaque. Those hindrances can be waived in part when one focuses on quasi-two-dimensional systems. In 2003, Klokkenburg et al. [6] succeeded in creating and characterizing the microstructure of thin layers of circa $40 \mathrm{~nm}$ in thickness using the cryogenic transmis-

\footnotetext{
${ }^{1}$ The article was translated by the authors.
}

sion electron microscopy technique (cryo-TEM). The diameter of the cores of the ferrofluid nanoparticles used by Klokkenburg et al. [6] ranged between 9 and $25 \mathrm{~nm}$, leading to systems that for practical purposes could be considered as quasi-two-dimensional (Q2D) systems, namely: particles are basically constrained to move in a plane while their dipoles are free to rotate and point in whatever direction of the three-dimensional space. The analysis of the snapshots taken by Klokkenburg et al. [6] showed that the magnetic nanoparticles confined in those thin nanolayers tend to form chain-like and ring-like structures.

From the theoretical point of view, the analysis of the microstructures found in bulky ferrofluids has received considerable attention in the last years [1013]. In the case of quasi-two-dimensional systems, despite some works have already shed some light on the microstructure of such systems, [7-9] and [1416], those systems are still poorly understood. Previous works have disclosed that the microstructure of magnetic fluids in thin layers differs considerably from the three-dimensional case. In the case of Q2D systems, the initial works have mainly focused on the study of monodisperse particles which are the easiest and most idealized systems. Nonetheless, real ferrofluids are polydisperse, i.e., their particles exhibit a certain distribution of sizes. Such distribution of particle sizes is commonly approximated by a gamma distribution. In the previous work [12] it was shown that bidisperse systems are usually good enough to account for the effects that polydispersity induces in a ferrofluid at a qualitative level: in this case, the continuous gamma distribution can be replaced by the two-stage diagram [17]. 
In this paper we study the microstructure of bidisperse ferrofluids in a thin layer (a quasi-two-dimensional geometry). The study of bidisperse systems represents a natural step forward in the study of Q2D ferrofluid systems once the microstructure of monodisperse systems has been quite fairly understood [7]. In this work a theoretical model for Q2D bidimensional systems based on the minimization of a free energy density functional is presented. This theoretical framework allows us to estimate the sizes, number and topology of the principal aggregates present in the thermodynamic equilibrium. The theoretical predictions of the model are stringently tested against the numerical results obtained via Langevin Dynamics simulations. The combination of theoretical study and computer simulations allows one to validate the model and get a much deeper understanding of the microstructure that magnetic nanocolloids exhibit in a thin layer.

In order to characterize ferrofluids one should note that in the case of monodisperse particles, all particles have the same magnetic core diameter $(\sigma)$ that is surrounded by a nonmagnetic organic thin layer with thickness $l$. All particles bear a magnetic moment $\boldsymbol{\mu}$ of the same strength. Therefore, in the monodisperse case, a single parameter is enough to characterize the effects of the magnetic dipole-dipole interaction: the dipolar coupling parameter is defined as

$$
\lambda=\frac{\mu_{0} \mu^{2}}{4 \pi k T(\sigma+2 l)^{3}},
$$

where $\mu_{0}=4 \pi \times 10^{-7} \mathrm{H} \mathrm{m}^{-1}$ is the vacuum permeability, $k$ is the Boltzmann constant and $T$ is the system temperature. The parameter $\lambda$ represents a comparison of the magnetic dipole-dipole interaction intensity $\mu_{0} \mu^{2} / 4 \pi(\sigma+2 l)^{3}$ to the thermal energy $k T$ for a pair of particles in close contact with their magnetic moments in a "head-to-tail" configuration.

Nonetheless, in bidisperse systems, due to the existence of two different kind of particles, the description of the magnetic interaction is more complex. Hereby, we will refer to the two kinds of particles as small and large particles using $\sigma_{s}$ and $\sigma_{l}$ to denote their respective diameters. In the same way, the magnetic moments of the two types of particles will be referred as $\boldsymbol{\mu}_{s}$ and $\boldsymbol{\mu}_{l}$. In a bidisperse systems, the two types of particles lead to three different types of coupling pairs: large-large, small-small and large-small. Therefore the description of a bidisperse system will require the introduction of three different coupling parameters:

$$
\lambda_{l l}=\frac{\mu_{0} \mu_{l}^{2}}{4 \pi k T\left(\sigma_{l}+2 l\right)^{3}}
$$

to describe the relative strength of the dipole-dipole interaction to the thermal forces in the case of two large particles,

$$
\lambda_{s s}=\frac{\mu_{0} \mu_{s}^{2}}{4 \pi k T\left(\sigma_{s}+2 l\right)^{3}}
$$

in the case of two small particles, and

$$
\lambda_{s l}=\frac{2 \mu_{0} \mu_{s} \mu_{l}}{\pi k T\left(\sigma_{s}+\sigma_{l}+4 l\right)^{3}}
$$

in the mixed case. The different types of interactions increase the complexity of the morphologies one can observe and hence the theoretical analysis one should perform.

In what follows, the details of the computer simulations for a system of dipole particles in a thin layer are described in Section 2. Section 3 is devoted to the development of the theoretical model for those bidisperse systems and to the investigation of the microstructures predicted by the model. The comparison and discussion of the theoretical predictions and computer simulations results are presented in Section 4. The main conclusions and results of this paper are reviewed in Section 5.

\section{SIMULATION METHOD}

Computer simulations have reached a state of the art mature enough to actively help in the investigation of complex physical, chemical and biological systems. In the study of Soft Matter, two main families of simulation methods exist: the first family includes those methods in which the phase space of the system is sampled through the evolution of some equations of motion, like for instance in the molecular dynamics technique [18]. The second family includes those methods in which the phase space is sampled in a stochastic way, like for instance in the Monte Carlo methods [18, 19]. Mixed techniques also exist like for instance the Langevin dynamics (LD) [7, 13]. In the LD technique the equations of motion are similar to those used in molecular dynamics but the solvent is taken into account in an implicit way using two forces: a stochastic force acting onto the particles cast in such a way that particles will mimic Brownian motion, and a friction force to take into account the effects of the viscosity of the solvent. In certain fields of research is also quite common to label Langevin dynamics with more generic names like molecular dynamics or stochastic dynamics. In any case, the physical equivalence between the families of methods relies on the ergodic hypothesis which states that the averaging of a certain observable over time is equivalent to its averaging over a representative statistical ensemble of the system.

LD has been the method chosen to perform the numerical simulations. We model the thin layer of ferrofluid as $M$ small and $N$ large spherical particles with magnetic core diameters $\sigma_{s}$ and $\sigma_{l}$, respectively. The 
centers of all particles are restricted to move inside a plane. Each particle possess a magnetic dipolar moment $\boldsymbol{\mu}_{s(l)}$ which can rotate not only in the plane of the monolayer, but in a complete three-dimensional space. The particles exhibit two different types of interactions: the first one is a point dipole-dipole interaction that mimics the magnetic interactions among the particles. The magnetic point dipoledipole interaction is

$$
U_{d d}(i, j)=\frac{\mu_{0}}{4 \pi}\left[\frac{\left(\boldsymbol{\mu}_{i} \cdot \boldsymbol{\mu}_{j}\right)}{\left|\mathbf{r}_{i j}\right|^{3}}-3 \frac{\left(\boldsymbol{\mu}_{i} \cdot \mathbf{r}_{i j}\right)\left(\boldsymbol{\mu}_{j} \cdot \mathbf{r}_{i j}\right)}{\left|\mathbf{r}_{i j}\right|^{5}}\right],
$$

where $\mathbf{r}_{i j}=\mathbf{r}_{i}-\mathbf{r}_{j}$ is a displacement vector connecting the centers of particles $i$ and $j$. In the case of ferrofluid particles we do not take into account Van der Waalslike interactions because those interactions are short ranged and they are effectively screened by the steric repulsion that the organic layers adsorbed onto the surface of the core of the particles. In fact, such organic layers have been added with the specific purpose of avoiding coagulation and render the colloidal suspension stable [6]. The second type of interaction is a soft core repulsion among particles that has been modeled as in previous works, [7, 13], using the Weeks-Chandler-Andersen potential (soft-sphere potential) [20]

$$
\begin{aligned}
& U_{\text {WCA }}(i, j) \\
& = \begin{cases}4 \varepsilon\left[\left(\frac{\sigma_{i}+\sigma_{j}}{2 r_{i j}}\right)^{12}-\left(\frac{\sigma_{i}+\sigma_{j}}{2 r_{i j}}\right)^{6}\right]+\varepsilon, & r_{i j}<r_{c}, \\
0, & r_{i j} \geq r_{c},\end{cases}
\end{aligned}
$$

which is the repulsive part of the Lennard-Jones potential with a cut-off set to $r_{c}=2^{-5 / 6}\left(\sigma_{i}+\sigma_{j}\right)$. The use of a coarse-grain model to study the $\mathrm{Q} 2 \mathrm{D}$ systems is justified because the size of the particles is large and each particle contains a number of atoms large enough as to be described accordingly to the laws of classical mechanics. As we will see in our results, there is no need to include in the model the atomistic details of the particles to mimic the behavior found in ferrofluid particles.

The initial conformation in each simulation is obtained by randomly distributing the centers of the particles inside a plane of side $L$. Magnetic moments directions are also distributed randomly and allowed to rotate freely without any restrictions in the threedimensional space. In order to take into account the long-range dipole-dipole interaction effectively and avoid unphysical behaviors due to the finite size effects, we use periodic boundary conditions (PBC) by replicating our main system cell along the plane. The introduction of periodic boundary conditions helps to minimize the finite size effects in simulations. Nonetheless, PBC adds an additional degree of complexity because now we have to take into account the longrange magnetic interactions of our particles with all the particles of the infinite replicas of the system along the plane.

When PBC are used, one of the fastest and more accurate algorithms to take into account the longrange, dipole-dipole interactions is the dipolar $\mathrm{P}^{3} \mathrm{M}$ algorithm $\left(\mathrm{dP}^{3} \mathrm{M}\right)$ [21]. That method assumes that periodic boundary conditions are taken along the three directions of the space. For that reason, in order to be dealing with Q2D systems, the fastest and most efficient way is to combine $\mathrm{dP}^{3} \mathrm{M}$ with the so-called dipolar layer correction (DLC) method [22] that allows to discount the contribution of the interactions due to particles which belong to planes different from the one that contains our original system cell.

The parameters of the $\mathrm{dP}^{3} \mathrm{M}$ and DLC methods has been tuned in order to execute the calculations as fast as possible with an accuracy in the calculus of the magnetic forces no less than $\delta \sim 10^{-4}$. The correctness of the implementation of the $\mathrm{dP}^{3} \mathrm{M}$ and DLC methods in our simulations has been tested by comparing their results against those obtained when using traditional Ewald summation methods [23, 24]. An important advantage of the combined use of $\mathrm{dP}^{3} \mathrm{M}$ and DLC respect of traditional Ewald sums adapted to the Q2D geometry, is that $\mathrm{dP}^{3} \mathrm{M}+\mathrm{DLC}$ do perform significantly faster in the calculation of the long-range interactions. Such advantage increases with the number of particles because the computer time needed to compute magnetic interactions scales like $(N+M) \ln (N+M)$ in the case of the $\mathrm{dP}^{3} \mathrm{M}+$ DLC method while in the case of Ewald sums the computer time scales like $(N+$ $M)^{3 / 2}$ even in the most optimized cases.

In our simulations we use an implicit solvent in order to avoid devoting most of the computer time to handle the massive amount of solvent particles one needs to include to simulate the solvent explicitly. In order to mimic the thermal motion that such solvent particles will induce on our ferrofluid particles, a Langevin thermostat is used. The viscosity effects due to the solvent are taken into account via a frictional force proportional to the velocity of the ferrofluid particles. Thus, the dynamics of our ferrofluid particles and their magnetic dipoles is governed by the following translational and rotational equations of motion [18]:

$$
\begin{aligned}
M_{i} \frac{d \mathbf{v}_{i}}{d t} & =\mathbf{F}_{i}-\Gamma_{T} \mathbf{v}_{i}+2 \xi_{i}^{T}, \\
I_{i} \frac{d \boldsymbol{\omega}_{i}}{d t} & =\boldsymbol{\tau}_{i}-\Gamma_{R} \boldsymbol{\omega}_{i}+2 \xi_{i}^{R},
\end{aligned}
$$

where $\mathbf{v}_{i}$ and $\boldsymbol{\omega}_{i}$ are the velocities of the translation and rotation of the $i$ th particle, respectively, and $\mathbf{F}_{i}$ and $\boldsymbol{\tau}_{i}$ are the force and torque acting on the particle $i$. $M_{i}$ and $I_{i}$ are the mass and inertia tensor of the particle, respectively. $\Gamma_{T}$ and $\Gamma_{R}$ are the translational and rotational friction coefficients. $\xi_{i}^{T}$ and $\xi_{i}^{R}$ represent the stochastic forces and torques which obey a Gaussian distribution (Einstein fluctuation-dissipation theo- 
rem) in order to mimic the effects of Brownian motion. All variables used in our computer simulations are dimensionless: distance $r^{*}=r / \sigma_{s}$, magnetic moment $\mu^{* 2}=\mu^{2} /\left(\varepsilon / \sigma_{s}^{3}\right)$, temperature $T^{*}=k_{\mathrm{B}} T / \varepsilon$ and time $t^{*}=t \sqrt{\varepsilon / M_{s} \sigma_{s}^{2}}$, where $M_{s}$ is the saturation magnetization for the investigated ferrofluid sample. All our simulations are performed at constant temperature $T^{*}=1$. Since we are not interested in the dynamical properties of the system but those properties corresponding to the thermal equilibrium state, we have some freedom in choosing the value of the mass, the inertia tensor, and the friction coefficients $\Gamma_{T}$ and $\Gamma_{R}$ of the particles. Therefore, in our simulations we have considered that the mass and the inertia tensor of the particles as equal to unity: $M_{i}=1$ and $I_{i}=1$ for each $i$.

The values of friction coefficients $\Gamma_{T}=1$ and $\Gamma_{R}=$ $3 / 4$ are selected in order to minimize the equilibration time needed to reach the equilibrium state. In our simulations the time step is set to $\Delta t^{*}=15 \times 10^{-4}$. All the systems are allowed to equilibrate for $2 \times 10^{6}$ time steps in order to warrant that no correlations exist with the initial condition and that the system has reached the equilibrium regime. In order to ensure that the system has reached the equilibrium state, several observables like the energy are monitored. The analysis shows that the typical correlation time among conformations does not exceed $10^{4} \Delta t^{*}$ even in the worst cases, therefore the ferrofluid microstructure analysis is performed during the next $8 \times 10^{6}$ time steps with measures taken at intervals of $2 \times 10^{4} \Delta t^{*}$ in order to sample uncorrelated states, and have good statistics.

The simulations are performed in systems in which the total number of particles is 1000 . Nonetheless, several tests with more than 10000 particles have been performed in order to check that results did not depend on the size of the system. All simulations have been performed using the simulation package ESPResSo [25].

In order to compare the results arising from numerical simulations with the theoretical predictions arising from the density functional theory (see next section), we need to determine the aggregates formed by the ferrofluid particles. In order to decide if two different particles do they belong to the same cluster or not, we use the so-called entropy criterion [13] that states that two particles will belong to the same cluster if the following three conditions are fulfilled:

$$
\left(\boldsymbol{\mu}_{i} \cdot \boldsymbol{\mu}_{j}\right) \geq 0, \quad\left(\boldsymbol{\mu}_{i} \cdot \mathbf{r}_{i j}\right)\left(\boldsymbol{\mu}_{j} \cdot \mathbf{r}_{i j}\right) \geq 0, \quad r_{i, j} \leq r_{c} .
$$

Thus, in order to belong to the same cluster the angles between the magnetic moments of the two particles have to be equal to or less than 90 degrees at the condition that the angles between the displacement vector and magnetic moments are either both acute, or both obtuse. At the same time, the distance between particles should be less than a certain cut-off distance $r_{c}$.
For a correct comparison of theory and simulation results, it is necessary to use in the analysis of both cases the same clustering criterion.

\section{THEORETICAL MODEL}

The magnetic moment of a particle is known to relax according to two different mechanisms depending on the size of the magnetic domains of the particles. In the case of small particles, with a upper limit size circa 5-6 $\mathrm{nm}$ for magnetite particles, the socalled Néel relaxation mechanism dominates. The Néel relaxation is characterized by a rotation of the dipole moment inside the crystalline lattice while the particle is fixed in space. In the case of larger particles the Brownian mechanism dominates: the dipole of the particle is fixed in the crystal lattice but it changes its orientation due to a rotation of the particle as a whole rigid body. The rotation of the particle is due to the solvent particles colliding with the colloidal particles (rotational Brownian motion). In our work we have chosen to study particles of size similar to those reported in the experimental works by Klokkenburg et al. [6] which are large enough to neglect the effects due to Néel relaxation mechanism and the only effect to take into account is Brownian relaxation.

In order to describe theoretically the Q2D bidisperse systems, we proceed in the same way as in the simulations: we consider small and large particles with magnetic cores of diameter $\sigma_{s}$ and $\sigma_{l}$ respectively, surrounded by a thin nonmagnetic layer of thickness $l$. The centers of all particles lie in a same plane. The magnetic moments are located in the centers of the particles and the interactions among particles are considered through the same pair-potentials than in the case of the numerical simulations, Eqs. (1) and (2).

Unfortunately, it is not possible in our theoretical framework to account for all the interactions of a particle with the rest of the particles of the system and their replicas because that would render our analytical calculations unfeasible. Thus, in our theoretical model we assume that the magnetic dipole-dipole interaction is only acting among first neighbors belonging to the same cluster. Furthermore, we assume that particles will only arrange to form flexible chain aggregates and quasi-ideal rings, and therefore in this first approach to the characterization of bidisperse systems we neglect the existence of complex branched structures [26, 27]. It is known that in difference to the theoretical description of bulky ferrofluids where it was enough to consider the fluid as an ideal gas of aggregates [12,13], in the case of Q2D ferrofluids it is important to account for the excluded area interactions in order to have a proper description of the system [7].

In a bidisperse system, the structure and the excluded area of a chain depend on the number of small and large particles that form the cluster as well as on their exact location in the chain sequence. Hence- 


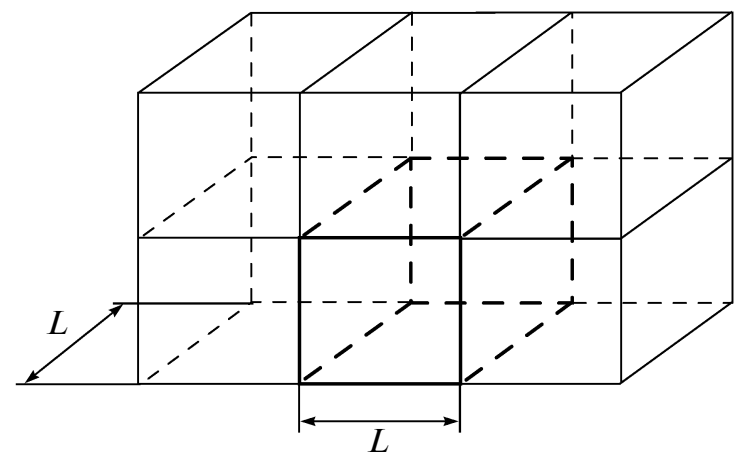

Fig. 1. Replication of the simulation box in the threedimensional space $L \times L \times L$. The main cell or simulation box is marked out by bold lines and its replicas are in thin lines.

forth, the area fraction of the chains corresponding to the same topological structure $\mathrm{i}$ and consisting of $n$ large particles and $m$ small particles will be denoted $g(i, n, m)$. In the case of quasi-ideal rings the centers of the particles form a regular $n$-gon which sides fluctuate in length. In this initial model, we assume that rings do only contain large particles. Results found in experiments [6] and computer simulations give strong support for this last assumption. Furthermore, rings whose particles are all large particles are expected to be the preferred state when $T \longrightarrow 0$, i.e. in the ground state $[15,16]$, and we will denote the area fraction associate to rings consisting of $n$ large particles as $f(n)$. Clusters composed of less than five particles cannot be properly classified as rings $[15,16]$, and we will always consider such small clusters as chains.

Once the theoretical model is specified, the first question one should tackle is how to obtain the specific number of rings and different topological chains that exist in a given Q2D bidisperse system composed of $N$ large and $M$ small particles. In order to answer that question the free energy density functional theory (DFT) will be used. The DFT methods are traditionally based on Frenkel heterophase fluctuations theory [28]. Kantorovich et al. [7] were successful in adapting the DFT to explain the case of a monodisperse Q2D ferrofluid. In the study of bidisperse systems, a combination of the theoretical framework developed in [7] and the algorithm introduced by Ivanov et al. [12] will be used. That approach allows to write the free energy density functional for a Q2D bidisperse system as:

$$
\begin{gathered}
F=k T \sum_{n+m \geq 1}^{\infty} \sum_{i=1}^{I(n, m)} K(i, n, m) g(i, n, m) \\
\times\left[\ln \left(\frac{g(i, n, m) s(i, n, m)}{e\left(1-S_{\mathrm{ex}} / S\right)}\right)-\ln Q(i, n, m)\right] \\
+k T \sum_{n=5}^{\infty} f(n)\left[\ln \left(\frac{f(n) s_{l}(n)}{e\left(1-S_{\mathrm{ex}} / S\right)}\right)-\ln W(n)\right],
\end{gathered}
$$

where $I(n, m)$ is the number of energetically distinguishable chains consisting of $n$ large and $m$ small particles. The number of all entropically distinguishable chains belonging to the $i$ th topological class, consisting of $n$ large and $m$ small particles, is determined by the combinatorial factor $K(i, n, m)$. The configuration integrals of chains and rings are denoted $Q(i, n, m)$ and $W(n)$ respectively. The calculation of $Q(i, n, m)$ and $W(n)$ depends on the cluster definition, because the integration in the space of particle positions and magnetic moment orientations has restrictions that depend on the type of cluster. The definition the different types of clusters will be done accordingly to the before mentioned entropy criterion.

Henceforth, $s(i, n, m)$ and $s_{l}(n)$ denote the area occupied by a chain or a ring, respectively. The area in which no particle external to the cluster can be placed will be denoted $S_{\mathrm{ex}}$. The areas $s(i, n, m)$ and $s_{l}(n)$ can be written in terms of the areas of the principal crosssections of large $s_{l}$ and small $s_{s}$ particles. The topological structure of the chains in these calculations can be taken into account by introducing the parameters determining the general number of bonds in a chain, thus: $a$ will denote the number of bonds between small-small particles, $b$ the number of particle bonds between small-large particles, and $c$ the number of bonds between large-large particles. Thus, and using previous notation, the areas $s(i, n, m)$ and $s_{l}(n)$ can be written as

$$
s(i, n, m)=s_{s}^{a} s_{s l}^{m-b} s_{l}^{n-c} \text { and } \quad s_{l}(n)=s_{l}^{n-1},
$$

where

$$
\begin{gathered}
s_{s}=\frac{\pi}{4}\left(\sigma_{s}+2 l\right)^{2}, \quad s_{s l}=\frac{\pi}{4}\left(\sigma_{s}+\sigma_{l}+4 l\right)^{2}, \\
s_{l}=\frac{\pi}{4}\left(\sigma_{l}+2 l\right)^{2} .
\end{gathered}
$$

In turn, the configuration integrals $Q(n, m)$ and $W(n)$ in the absence of an external magnetic field and if we restrict the interactions to nearest neighbors, can be written as

$$
\begin{gathered}
Q(n, m)=q_{0}\left(\lambda_{11}\right)^{a} q_{0}\left(\lambda_{12}\right)^{b} q_{0}\left(\lambda_{22}\right)^{c}, \\
W(n)=\frac{1}{n} w_{0}^{n}(n),
\end{gathered}
$$

where $q_{0}\left(\lambda_{i j}\right)$ and $w_{0}(n)$ are the partition functions of a dimer that forms part of chain or a ring, respectively. Expressions for $q_{0}\left(\lambda_{i j}\right)$ and $w_{0}(n)$ are derived in [7]. To simplify further analysis, we also introduce the bond energy vector $\mathbf{E}=\left(e_{11}, e_{12}, e_{22}\right)$, where $e_{i j}=\ln \left(q_{0}\left(\lambda_{i j}\right)\right)$.

In the expression for the free energy density functional (6), the sum of the free energies of all chains and rings are described by the first and second term, respectively. Those terms take into account the energy and entropy of the aggregates. Preliminary results have shown that it is very important to take into account the excluded area $S_{\mathrm{ex}}$. Due to the fact that in our bidisperse systems the chain aggregates can be formed by large 


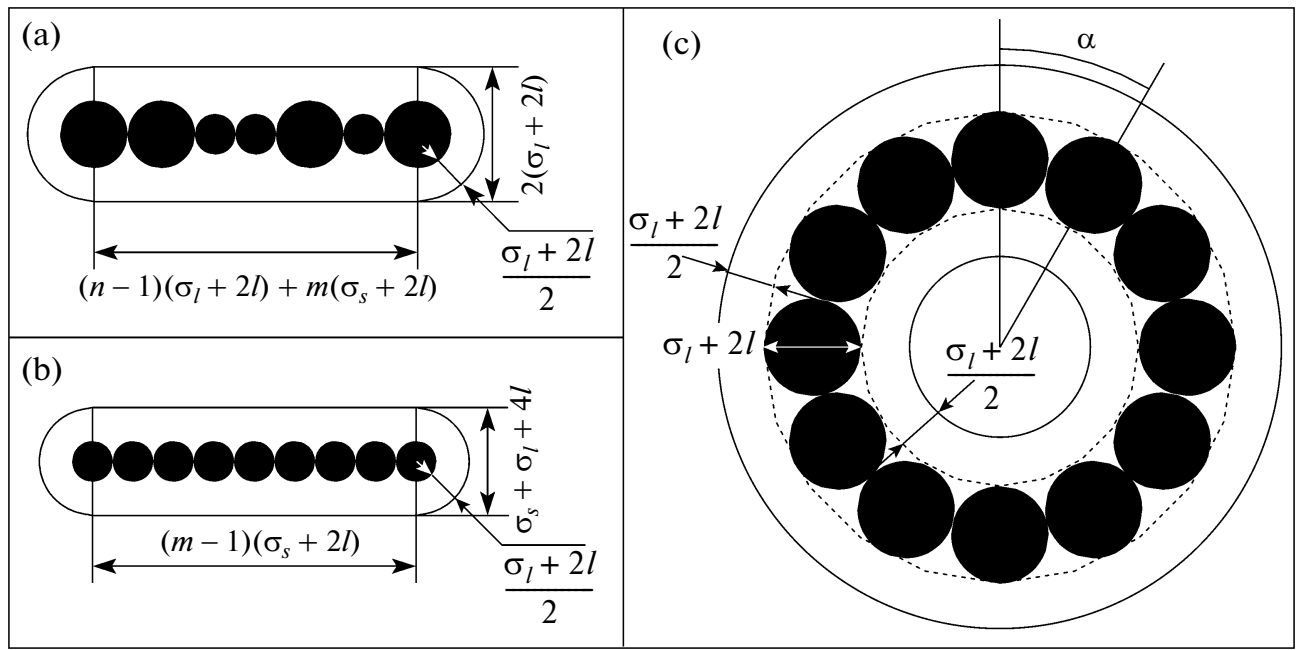

Fig. 2. Excluded areas: (a) for the chains consisting of $n$ large and $m$ small particles; (b) for the chains consisting of $m$ small particles only; (c) for the rings, consisting of $n$ large particles. One should remark that the theory allows for fluctuations of the particles that form part of the cluster as far as entropy criterion is satisfied. In all figures in this work chains and rings are depicted as ideal clusters for the sake of illustration clarity.

and small particles, while rings are assumed to be only composed of large particles, it will be necessary to calculate the excluded area for chains and rings separately. As an example, Fig. 2 depicts the excluded areas corresponding to two different possible types of chains and a ring. It will be necessary to consider separately chains which are formed only by large particles from those with a mixed composition (see Fig. 2a), as well as from chains only composed of small particles (see Fig. 2b), because each of them gives a different contribution to the general formula for the excluded area.

In general, the excluded area of a chain consisting of an arbitrary number of large particles $(n \geq 1)$ and an arbitrary number of small particles $(m \geq 0)$, that would correspond to case (a) in Fig. 2, can be thought as the sum of the area of the rectangle with sides $(n-1) \sigma_{l}+$ $m \sigma_{s}$ and $2 \sigma_{l}$ and the area of a circle with radius $\sigma_{l}$ :

$$
\begin{gathered}
S_{\mathrm{ex}}^{(a)}=2 \sigma_{l}\left[(n-1) \sigma_{l}+m \sigma_{s}\right]+\pi \sigma_{l}^{2} \\
=2 n \sigma_{l}^{2}+(\pi-2) \sigma_{l}^{2}+2 m \sigma_{1} \sigma_{s} .
\end{gathered}
$$

One should note that for calculating the excluded area in those mixed chains, the relative positions of large and small particles does not matter.

In the case of a chain composed exclusively by small particles, case (b) in Fig. 2, i.e. $m \geq 1$ and $n=0$, the excluded area is the sum of the rectangle area with sides $(m-1) \sigma_{s}$ and $\sigma_{l}+\sigma_{s}$ and the circle with radius $\left(\sigma_{l}+\sigma_{s}\right) / 2$ :

$$
S_{\mathrm{ex}}^{(b)}=(m-1) \sigma_{s}\left(\sigma_{l}+\sigma_{s}\right)+\pi \frac{\left(\sigma_{l}+\sigma_{s}\right)^{2}}{4} .
$$

On the other hand, for a ring with $n \geq 5$ large particles, case (c) in Fig. 2, the excluded area can be written as

$$
S_{\mathrm{ex}}^{(c)}=\frac{2 \pi \sigma_{l}^{2}}{\sin (\alpha / 2)} \approx 2 n \sigma_{l}^{2}, \quad \alpha=\frac{2 \pi}{n}, \quad n \gg 1 .
$$

Thus, when the contribution of all kinds of clusters and topologies is taken into account, the general expression for the excluded area is

$$
\begin{gathered}
S_{\mathrm{ex}}=S_{\mathrm{ex}}^{(a)}+S_{\mathrm{ex}}^{(b)}+S_{\mathrm{ex}}^{(c)} \\
=\sum_{n=1}^{\infty} \sum_{m=0}^{\infty} \sum_{i}\left[2 n \sigma_{l}^{2}+(\pi-2) \sigma_{l}^{2}+2 m \sigma_{l} \sigma_{s}\right] \\
\times \tilde{g}(n, m, i)+\sum_{m=1}^{\infty}\left[(m-1) \sigma_{s}\left(\sigma_{l}+\sigma_{s}\right)+\pi \frac{\left(\sigma_{l}+\sigma_{s}\right)^{2}}{4}\right] \\
\times \tilde{g}(0, m)+\sum_{n=5}^{\infty} 2 n \sigma_{l}^{2} \tilde{f}(n),
\end{gathered}
$$

where $\tilde{g}(n, m, i), \tilde{g}(0, m)$, and $\tilde{f}(n)$ are the numbers of chains and rings of the same length, i.e. containing the same number of particles. The corresponding area fractions are

$$
\begin{gathered}
g(n, m, i)=\frac{\tilde{g}(n, m, i)}{S}, \quad g(0, m)=\frac{\tilde{g}(0, m)}{S}, \\
\quad \text { and } f(n)=\frac{\tilde{f}(n)}{S} .
\end{gathered}
$$


Dividing and multiplying each of the terms in Eq. (11) by the area of the sample $S$ we get:

$$
\begin{gathered}
S_{\mathrm{ex}}=S\left[\sum_{n=1}^{\infty} \sum_{m=0}^{\infty} \sum_{i} \frac{2 n}{S} \sigma_{l}^{2} \tilde{g}(n, m, i)+\sum_{n=5}^{\infty} \frac{2 n}{S} \sigma_{l}^{2} \tilde{f}(n)\right] \\
+S\left[\sum_{n=1}^{\infty} \sum_{m=0}^{\infty} \sum_{i}\left(\frac{\pi-2}{S} \sigma_{l}^{2}+\frac{2 m}{S} \sigma_{l} \sigma_{s}\right) \tilde{g}(n, m, i)\right. \\
+\sum_{m=1}^{\infty}\left(\frac{(m-1)}{S} \sigma_{s}\left(\sigma_{l}+\sigma_{s}\right)\right. \\
\left.\left.+\frac{\pi}{S} \frac{\left(\sigma_{l}+\sigma_{s}\right)^{2}}{4}\right) \tilde{g}(0, m)\right]
\end{gathered}
$$

Note that

$$
\sum_{n=1}^{\infty} \sum_{m=0}^{\infty} \sum_{i} n \tilde{g}(n, m, i)+\sum_{n=5}^{\infty} \tilde{n f}(n)=N
$$

amounts to the number of large particles in the system, and

$$
\sum_{n=0}^{\infty} \sum_{m=1}^{\infty} \sum_{i} m \tilde{g}(n, m, i)=M
$$

is the total number of small particles in the system.

It is also known that area fractions corresponding to large and small particles in the system are $\phi_{l}=N s_{l} / S$ and $\phi_{s}=M s_{s} / S$ respectively. Finally, the excluded area can be expressed in the following way:

$$
S_{\mathrm{ex}}=S\left(\frac{8}{\pi} \phi_{l}+4 \beta \phi_{s}\right) .
$$

Here, $\beta \ll 1$ is a small parameter replacing the remaining terms. This factor can be calculated directly, but its calculation is cumbersome and it is known to be an almost vanishing quantity. Therefore, we have set $\beta=$ 0.01 for the systems with large particles of diameter $d_{l}=20 \mathrm{~nm}$ and $\beta=0.05$ for the systems with large particles of diameter $d_{l}=18 \mathrm{~nm}$.

In order to minimize the free energy density functional (6), we need to consider the natural balance conditions:

$$
\begin{gathered}
\frac{\phi_{l}}{s_{l}}=\sum_{n+m \geq 1}^{\infty} \sum_{i=1}^{I(n, m)} K(i, n, m) g(i, n, m) n \\
+\sum_{n=5}^{\infty} f(n) n, \\
\frac{\phi_{s}}{s_{s}}=\sum_{n+m \geq 1}^{\infty} \sum_{i=1}^{I(n, m)} K(i, n, m) g(i, n, m) m,
\end{gathered}
$$

where $\phi_{s}$ and $\phi_{l}$ are the surface densities of small and large particles of the system, respectively. These conditions ensure that the number of particles in the system will remain constant. Once the balance conditions are set, the method of Lagrange multipliers can be used to write the general form of the functional $\Phi$ :

$$
\begin{gathered}
\Phi=\frac{F}{k T}+v_{1}\left(\frac{\phi_{l}}{s_{l}}\right. \\
\left.-\sum_{n+m \geq 1}^{\infty} \sum_{i=1}^{I(n, m)} K(i, n, m) g(i, n, m) n+\sum_{n=5}^{\infty} f(n) n\right) \\
+v_{2}\left(\frac{\phi_{s}}{s_{s}}-\sum_{n+m \geq 1}^{\infty} \sum_{i=1}^{I(n, m)} K(i, n, m) g(i, n, m) m\right),
\end{gathered}
$$

where $v_{1}$ and $v_{2}$ are the Lagrange multipliers to be found from the system of equations:

$$
\begin{gathered}
\frac{\partial \Phi}{\partial g(i, n, m)}=0, \quad \forall n, m, \\
\frac{\partial \Phi}{\partial f(n)}=0, \quad \forall n, \\
\frac{\partial \Phi}{\partial v_{1}}=0, \\
\frac{\partial \Phi}{\partial v_{2}}=0 .
\end{gathered}
$$

Differentiating the functional $\Phi$ with respect to variables $g(i, m, n)$ for all $n$ and $m$, with respect to variables $f(n)$ for all $n$, and also with respect to variables $v_{1}$ and $v_{2}$, system of equations (17) can be written as:

$$
\begin{gathered}
\ln \frac{g(i, n, m) s(i, n, m)}{e\left(1-S_{\mathrm{ex}} / S\right)}-\left(a e_{11}+b e_{12}+c e_{22}\right) \\
-v_{1} m-v_{2} n+1=0, \\
\forall m, n: n+m \geq 1, \quad i=1, \ldots, I(n, m), \\
\ln \frac{f(n) s(n)}{e\left(1-S_{\mathrm{ex}} / S\right)}-\ln W(n)-v_{2} n+1=0, \\
\forall n: n \geq 5, \\
\frac{\phi_{l}}{s_{l}}=\sum_{n+m \geq 1}^{\infty} \sum_{i=1}^{I(n, m)} K(i, n, m) g(i, n, m) n \\
\quad+\sum_{n=5}^{\infty} f(n) n, \\
\frac{\phi_{s}}{s_{s}}=\sum_{n+m \geq 1}^{\infty} \sum_{i=1}^{I(n, m)} K(i, n, m) g(i, n, m) m .
\end{gathered}
$$

The first and second sets of equations in (18) allow to express equilibrium concentrations of chain aggre- 
gates $g(i, n, m)$ and rings $f(n)$ as the functions of Lagrange multipliers $v_{1}$ and $v_{2}$ :

$$
\begin{gathered}
g(i, n, m)=\left(1-S_{\mathrm{ex}} / S\right) \\
\times \frac{\exp \left(v_{1} m+v_{2} n+\left(a e_{11}+b e_{12}+c e_{22}\right)\right)}{s(i, n, m)}, \\
\forall m, n: n+m \geq 1, \quad i=1, \ldots, I(n, m), \\
f(n)=\left(1-S_{\mathrm{ex}} / S\right) \frac{\exp \left(n v_{2}\right) w_{0}^{n}}{s(n)}, \quad \forall n: n \geq 5, \\
\frac{\phi_{l}}{s_{l}}=\sum_{n+m \geq 1}^{\infty} \sum_{i=1}^{I(n, m)} K(i, n, m) g(i, n, m) n \\
\frac{\phi_{s}}{s_{s}}=\sum_{n+m \geq 1}^{\infty} \sum_{i=1}^{\infty} K(i, n, m) g(i, n, m) m .
\end{gathered}
$$

Note that the two last equations in system (19) represent derivatives with respect to Lagrange multipliers $v_{1}$ and $v_{2}$ that equal to zero, and are in fact the balance conditions (14) and (15). Unfortunately, the analytical summation of the series is impossible due to the presence of rings, and Lagrange multipliers must be calculated numerically. In the summation related to chains, it is convenient to sum up analytically all the contributions from chains which have different lengths but correspond to the same topological structure $i$. Here again, we make an approach by taking into account only the most representative chain topologies or structures that contribute to the microstructure of a bidisperse ferrofluid. Thus, only nineteen classes of different chain structures (see Fig. 3) have been taken into account in the model: cles;

- the chains composed exclusively of large parti-

- the chain aggregates that contain some large particles and only one small particle;

- the chains that contain some large particles and two small particles;

- the chain aggregates that contain several large particles and three small particles;

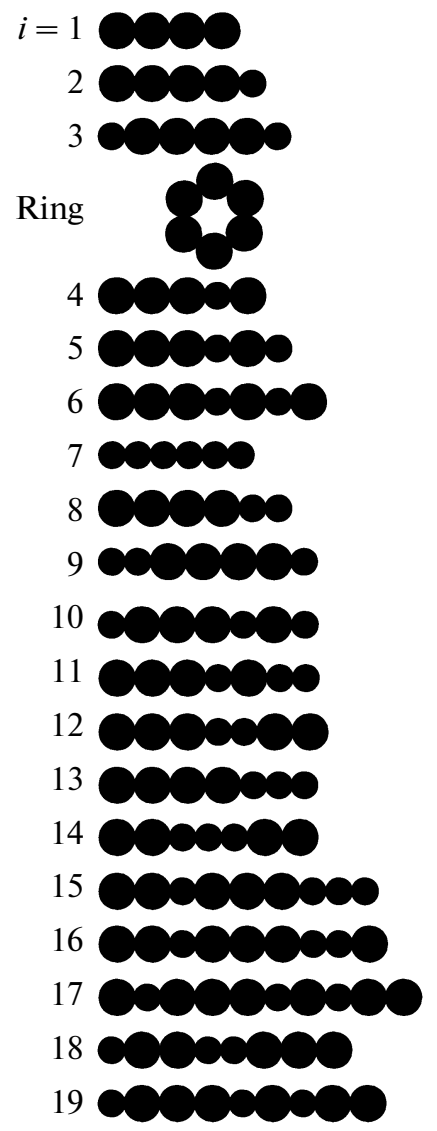

Fig. 3. Classes of clusters used in the theoretical analysis of the microstructure of a bidisperse ferrofluid in a quasi-twodimensional geometry.

- the chains containing several large particles and four small ones;

- the chain aggregates composed exclusively of small particles.

The contributions in series (14) and (15), as well as the equilibrium concentrations were calculated by introducing $p_{1}$ and $p_{2}$. The values of $p_{1}$ and $p_{2}$ are defined by Lagrange multipliers $v_{1}$ and $v_{2}$ (see their detailed expression (20) in Appendix), and represent non-normalized thermodynamic probabilities of forming a small-large and a large-large particle pair

Table 1. Basic characteristics of particles we used for modelling bidisperse ferrofluids in a quasi-two-dimensional geometry

\begin{tabular}{c|c|c|c|c}
\hline Fraction type & $\begin{array}{c}\text { Particle } \\
\text { designation }\end{array}$ & $\begin{array}{c}\text { Particle diameter, } \\
\sigma+2 l, \mathrm{~nm}\end{array}$ & $\begin{array}{c}\text { Magnetic nuclear } \\
\text { diameter, } \sigma, \text { nm }\end{array}$ & $\begin{array}{c}\text { Magnetic dipole-dipole } \\
\text { interaction parameter, } \lambda\end{array}$ \\
\hline \multirow{2}{*}{ Large particles } & A & 20 & 16 & 3.276 \\
& B & 18 & 14 & 2.017 \\
\hline \multirow{3}{*}{ Small particles } & C & 17 & 13 & 1.535 \\
& D & 15 & 9 & 0.819 \\
& E & 13 & 7 & 0.1378 \\
\hline
\end{tabular}



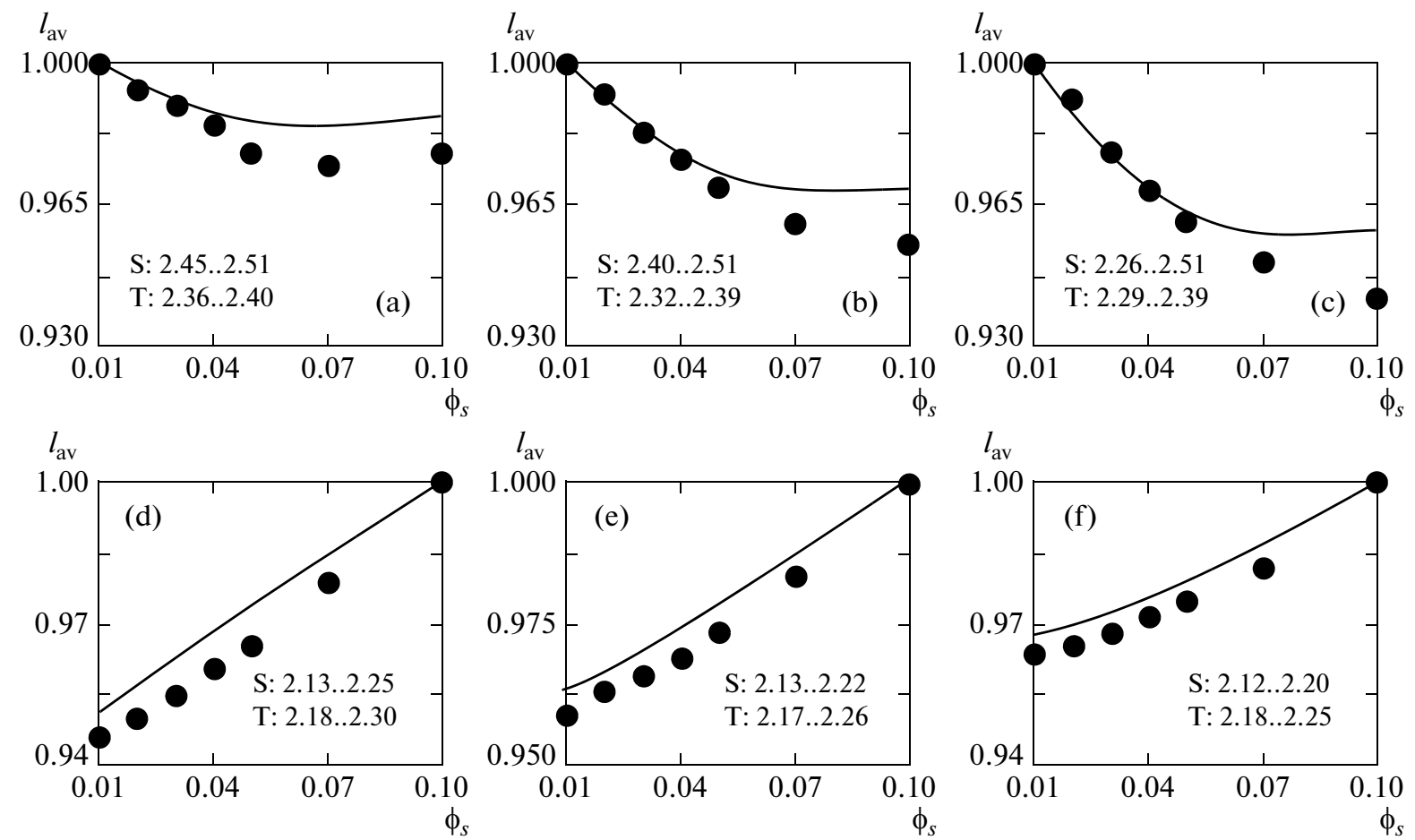

Fig. 4. Average chain length for the systems with large particles of $A$ and $B$ types as a function of small particle area fraction, normalized by its maximum value: (a) for the system $\mathrm{AD}\left(\sigma_{l}=16, \sigma_{s}=11\right)$, (b) for the system $\mathrm{AE}\left(\sigma_{l}=16, \sigma_{s}=9\right)$, (c) for the system $\operatorname{AF}\left(\sigma_{l}=16, \sigma_{s}=7\right)$, (d) is the system BD $\left(\sigma_{l}=14, \sigma_{s}=11\right),(\mathrm{e})$ is the system BE $\left(\sigma_{l}=14, \sigma_{s}=9\right)$, (f) is the system BF $\left(\sigma_{l}=14\right.$, $\left.\sigma_{s}=7\right)$. The solid lines are the theoretical results, dots are the computer simulation data. The insets in the plots show the range of variation of the non-normalized average chain length: T corresponds to the theory, S corresponds to computer simulations.

respectively. Therefore, it is sufficient to define the probabilities for finding equilibrium area fractions of aggregates. One can obtain those probabilities by using the two last equations of the system (19). Substituting in series all contributions calculated above, we obtain a system consisting of two equations with two variables $p_{1}$ and $p_{2}$. The solution of that system can be easily found numerically.

Thus, once probabilities $p_{1}$ and $p_{2}$ have been defined, the calculation of equilibrium concentrations of each possible chain structure and rings can be determined. In the next section, we present the results of those calculations and their comparison to the computer simulation data.

\section{RESULTS AND DISCUSSIONS}

In our study we have considered six types of particles of different size, covered by a thin nonmagnetic layer of thickness $l=2 \mathrm{~nm}$. The diameters of the particles and their magnetic core, as well as the magnetic dipole-dipole interaction parameters $\lambda$ for each kind of particle used in this work are collected in Table 1. We have considered particles of classes $A$ and $B$ as large particles, while the particles of classes C, D, E, and F have been considered as small particles. The bidisperse systems has been generated by combining particles belonging to a class of the large particles with particles of a class belonging to the small particles. This has lead to the thorough analysis of eight different bidisperse systems.

In our model, the temperature of the analyzed systems was constant and set to $T=293 \mathrm{~K}$. Area fractions of large particles A and B were identical $\phi_{l}=0.05$, while area fractions of small particles $\mathrm{C}-\mathrm{F}$ varied: $\phi_{s}=$ $0.01,0.02,0.03,0.04,0.05,0.07,0.1$.

In this work, we have focused on several properties of the bidisperse systems: the average aggregate length (size) $l_{\mathrm{av}}$ (average number of particles in the aggregate), area fractions of the aggregates, and the most probable aggregate topology. The average chain length as a function of the small particle area fraction for the systems $\mathrm{AD}, \mathrm{AE}, \mathrm{AF}, \mathrm{BD}, \mathrm{BE}, \mathrm{BF}$ is shown in Fig. 4. In the plots, the average chain length is normalized by the maximum value for each system to demonstrate the influence of the ratio of the particle diameters. In this way one can see that the largest change of the average chain length with increasing small particle area fraction is observed for systems AF and BD. The good agreement between theoretical estimations and results from numerical simulations allows to conclude that the developed theoretical model is adequate and applicable to describe the microstructure of a bidisperse ferrofluid in a thin layer. In the remaining systems, 
comparison of the average chain lengths in theory and simulations shows a similar agreement and, therefore, they are not plotted.

Our analysis shows that mainly short chains consisting of two-three particles are formed in the studied bidisperse systems. The length of the chains practically does not change when the area fraction of small particles increases. That differs qualitatively from a bulk bidisperse magnetic fluid, where the average chain length decreases considerably with the increase in the concentration of small particles [13]. The latter effect corresponds to the so-called "poisoning" effect [12]: the growth of chains is inhibited by the small particles sticking to the ends of the large-particle chains. In bulk, moreover, the probability of finding a chain with a small particle between two large ones is extremely low. In contrast, in a thin layer both large and small particles, are more or less participating in the chain formation. The increase of the small particle area fraction exerts a very weak influence on the ferrofluid microstructure in a thin layer. Despite the average chain length in a monolayer is found to be rather small (for the systems studied here), one should note that long chains with various topological compositions are still present in the bidisperse Q2D systems. Therefore, considering them in our theoretical description (see Fig. 3) turns out to be crucial.

In [13] it was shown that the microstructure of three-dimensional bidisperse ferrofluids could be characterized via chain aggregates belonging to the first three classes depicted in Fig. 3 plus single small particles. At this point, a rather natural question arises: how can one justify the need of up to 19 different topological classes in a monolayer? The key point is, that our preliminary studies showed that considering in a monolayer only the three classes we considered in bulk led the model to serious discrepancies with the results from simulations for all the range of parameters. In order to provide an idea of how big the disagreement is when a very reduced number of classes is taken into account, we depict in Fig. 5 the average chain length versus the area fraction of small particles that would correspond to the AE system if only the three main classes (see Fig. 3) and single small particles were taken into account to obtain the theoretical estimates. As Fig. 5 reveals, the theory and computer simulations results do not agree even qualitatively when so few morphologies are included in the model. However, as it was shown before in Fig. 4, if we take into account the nineteen classes of chains, the theoretical predictions and the computer simulation results show a very reasonable agreement. The need of including more morphologies in the theoretical model reveals that the presence of the geometric constraints leads to a qualitative change in the microstructure: small particles in the quasi-two-dimensional system cannot prevent the growth of the chains by sticking to the large ones. In other words, the "poisoning" effect being very pronounced in bulk, is damped by the geometrical con-

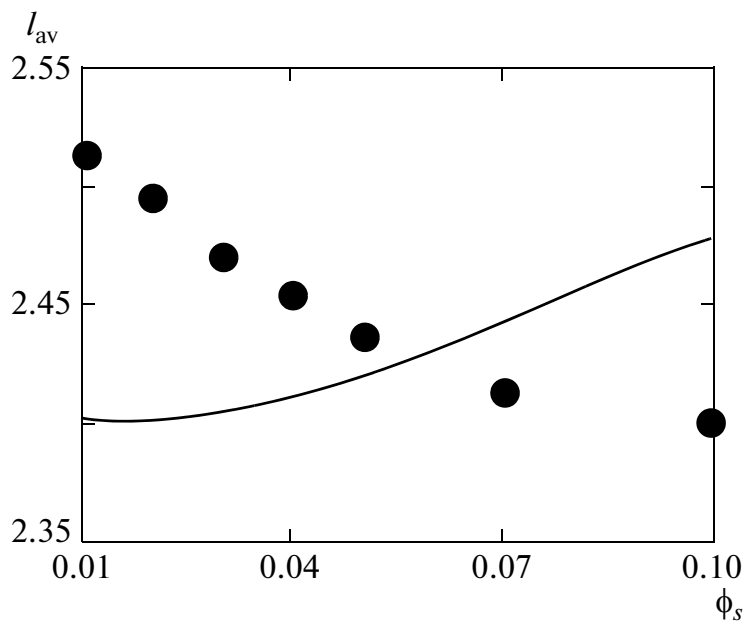

Fig. 5. Average chain length as a function of the small particle area fraction for the system $\operatorname{AE}\left(\sigma_{l}=16, \sigma_{s}=9\right)$ in a thin layer when only the first three chain classes (see Fig. 3), single small particles and rings are considered in the free energy density minimization (solid lines). Note that in simulations, no assumptions about the existing types of structures were done (dots). A strong qualitative disagreement between theory and computer simulation results indicates that it is necessary to take into account more classes in the theoretical estimation in order to describe the microstructure of a thin bidisperse layer.

straints in Q2D system. As a result, the microstructure of Q2D magnetic fluid films is far more complex than the one observe in bulk.

In order to understand what is the role of small particles in the aggregation process, we have studied the probability of forming chains, containing a certain number of small particles (from zero up to three). We focus on the dependence of the latter probability on the number of large particles in a chain. Here, we allow for all types of chain structures without distinguishing among topologies. Thus, for example, for calculating the probability of finding a chain containing one small particle, the chains of the second and fourth topological classes (see Fig. 3) are to be considered; for calculating the probability of finding a chain with two small particles we have to take into account four different chain classes: the third, fifth, sixth, and eighth from Fig. 3. The plots of these probabilities for the system BD with area fractions for the small particles of 0.01 and 0.1 are given in Fig. 6. Note that here we provide this plot only for one system as the behavior does not change qualitatively by changing the ratio of particle diameters. The agreement between theory and simulations is fairly good for all systems.

Obviously, the probability of forming chains with one, two and three small particles decreases with increasing the number of large particles in a chain. In most cases the decrease is characterized by the exponential law $g(i, n, m) / N_{\mathrm{ch}}$, where $N_{\mathrm{ch}}$ is the number of all the chains formed in the system under study. Looking at Fig. 6a, where the area fraction of small particles 


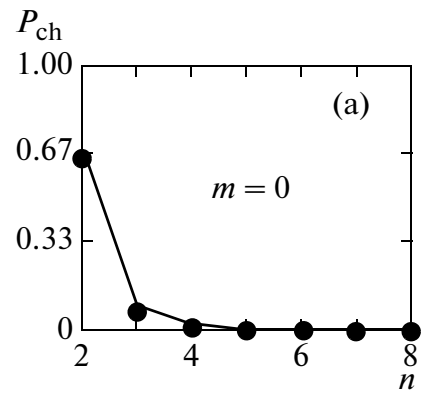

$P_{\text {ch }} \times 10^{3}$

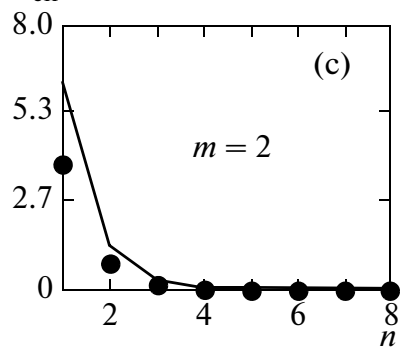

$$
P_{\text {ch }}
$$

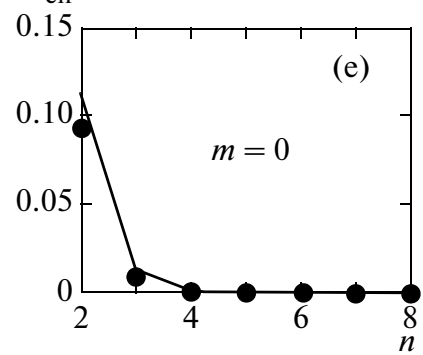

$P_{\mathrm{ch}}$

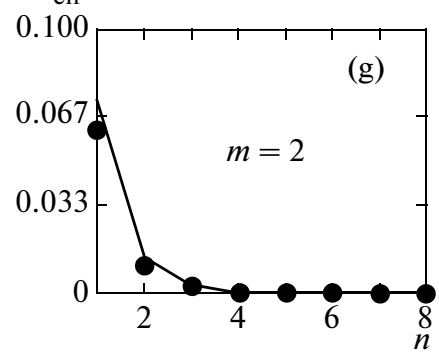

(g)

$P_{\mathrm{ch}}$

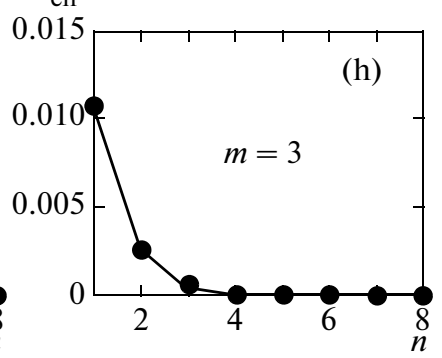

Fig. 6. Probabilities of forming chains with a defined number $m$ of small particles for the system BD $\left(\sigma_{l}=14, \sigma_{s}=\right.$ 11 ) as a function of the number $n$ of large particles in the structure. The area fraction of small particles in the system was equal 0.01 (a) $-(\mathrm{d})$ and 0.1 (e)-(h). Solid lines are the theory results, dots correspond to the results from the computer simulations.

is 0.01 , and comparing it to the plots in Figs. $6 b-6 d$ at the same area fraction, we conclude, that the most probable chain structure is the one consisting of two large particles only. The size of the dominant structure remains the same when the area fraction of small particles changes by one order of magnitude, however, the dimer composition changes: one large particle is replaced by a small one (see Fig. 6f). Here, one needs to underline, that the large particle dimer is energetically more advantageous. Another important conclusion here is that the probability of forming chains composed by exclusively small particles increases (comparison of plots in Fig. 6d and Fig. 6h) with increasing the small particle area fraction, which shows the active participation of small particles in the aggregation process. Thus, in contrast to bulk bidisperse ferrofluids, the chains, containing small particles in direct contact, will be present in the Q2D with a non-vanishing probability.

In order to find the reason why the microstructure in quasi-two-dimensional case is more complex in comparison to the one in bulk, we have studied the probability of forming large-large and large-small particle pairs for both situations. Taking into account the fact that the microstructure for a three-dimensional system is fairly described by the three first chain classes [13] plus the single small particles, we assumed that for fair comparison, only these structures have to be considered in quasi-two-dimensional system. In mathematical terms it means that it is necessary to estimate the rate of convergence of the series in the system of equations (19). The fact that more terms have to be considered in quasi-two-dimensional systems indicates that the number of significant contributions (whose absolute values are comparable) is larger in Q2D than in a three-dimensional system, where the first four terms are enough to ensure the convergence. In the analysis, it is also necessary to calculate volume concentrations of particles for both fractions in order for them to correspond to the area fractions we consider in Q2D. Values of these concentrations are given in Table 2 for small and large particles. Values of nonnormalized probabilities of forming large-small $p_{1}$ and large-large $p_{2}$ particle pairs are calculated while minimizing the free energy density functional (6):

$$
p_{1}=\exp \left(v_{1}+e_{12}\right), \quad p_{2}=\exp \left(v_{2}+e_{22}\right),
$$

where $v_{1}$ and $v_{2}$ are Lagrange multipliers, $e_{12}$ and $e_{22}$ are large-small and large-large particle pair energies respectively. For the sake of clarity, we introduce here the bond energy vector $\mathbf{E}=\left(e_{11}, e_{12}, e_{22}\right)$, where $e_{i, j}=$ $\ln \left(q_{0}(i j)\right)$. It is necessary to note that these energies are

Table 2. Area fraction of small particles for quasi-two-dimensional geometry and appropriate concentrations in threedimensional case

\begin{tabular}{c|c|c|c|c|c|c|c|c||c|c}
\hline$\phi_{s}^{2 D}$ & 0.01 & 0.02 & 0.03 & 0.04 & 0.05 & 0.06 & 0.07 & 0.1 & $\phi_{l}^{2 D}$ & 0.05 \\
$\phi_{s}^{3 D}$ & 0.007 & 0.013 & 0.020 & 0.027 & 0.033 & 0.040 & 0.047 & 0.067 & $\phi_{l}^{3 D}$ & 0.033 \\
\hline
\end{tabular}


smaller in Q2D than those in bulk [7]. To derive normalized probabilities we use the following definitions:

$$
P_{1}=\frac{p_{1}}{p_{1}+p_{2}}, \quad P_{2}=\frac{p_{2}}{p_{1}+p_{2}} .
$$

These probabilities in Q2D and bulk for the systems with large particles of type A and for all types of small particles are shown in Fig. 7. Due to the absence of qualitative differences, systems with large particles of type B are not provided here. All the plots exhibit similar qualitative behavior for both sample geometries and for each type of small particles: the probability of forming a large-small particle pair increases with the increase of the area fraction of small particles, while the one of forming a large-large particle pair decreases. However, in Q2D, for practically all systems, the probability of forming a large-small particle pair is higher than the one in bulk. On the contrary, the probability of forming a large-large particle pair is almost always lower in Q2D. This drives us to the following conclusion: under the influence of geometrical constraints the formation of clusters is mainly determined by entropy, whereas in bulky magnetic fluids the microstructure is energy-dictated. As a consequence, the spectrum of formed clusters in Q2D is noticeably wider, than the one in bulk. This conclusion is in fact nontrivial also because the effective energies of interaction are lower in Q2D. Importantly, the microstructure of a bidisperse ferrofluid is very sensitive not only to the granulometric composition, but also to the particle size ratio. Thus, for example, in Fig. $7 \mathrm{~d}$ and $7 \mathrm{~h}$, the probabilities have the opposite tendency. In other words, if small particles are too small in comparison to the large ones, then both in Q2D and bulk, the chain formation is determined by the energy, as the effective energy for large-small particle pair is negligible, and the large-large particle pair becomes dominant.

To summarize, our investigations showed that the microstructure of a bidisperse ferrofluid in a nanolayer is significantly more versatile than the one in bulk; its accurate description demands for considering nineteen topological classes of chains and rings.

\section{CONCLUSIONS}

In this work, combining analytical and simulations approaches, we have determined the microstructure of bidisperse monolayers of ferrofluid in the absence of external magnetic fields. Our results have allowed us to elucidate the differences induced by geometrical constraints. We have developed an efficient theoretical model for a bidisperse ferrofluid, based on the free energy density functional minimization. Results from the theoretical model and the simulations are in very good agreement. The substantial agreement means on the one hand that our theoretical model provides an adequate description of aggregation in bidisperse ferrofluids in a thin layer. On the other hand, it means,

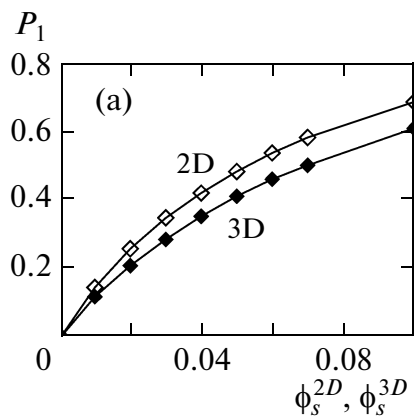

$P_{1}$
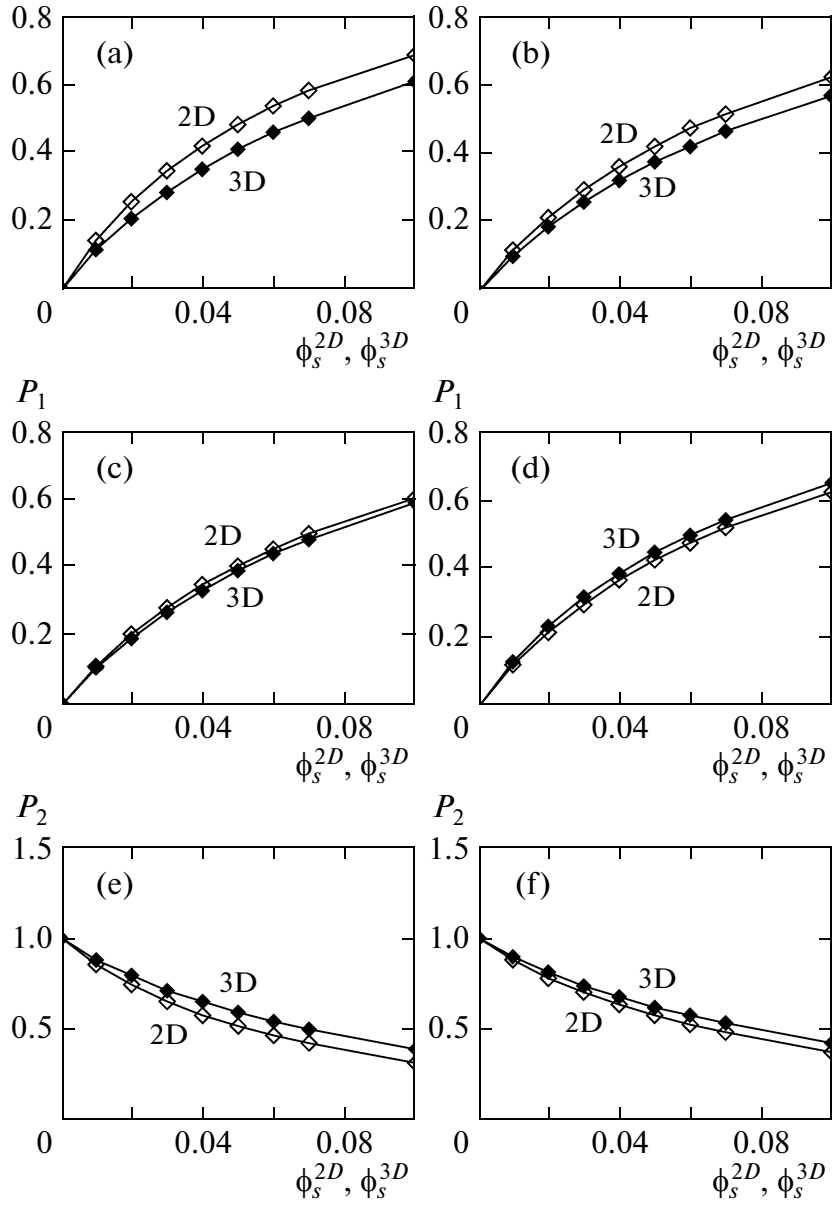

$P_{1}$

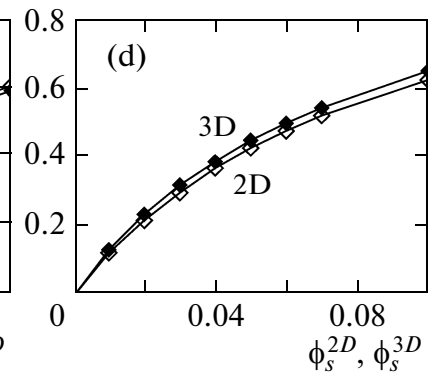

$P_{2}$
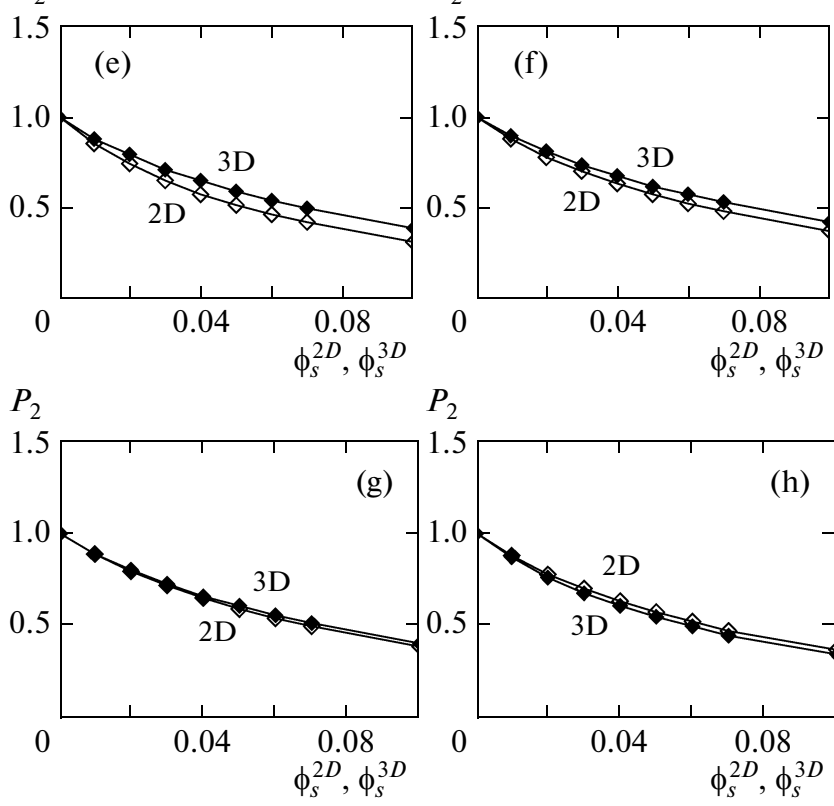

$P_{2}$

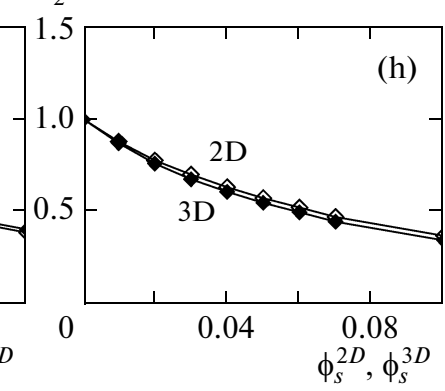

Fig. 7. Probabilities of forming large-small (a)-(d) and large-large (e)-(h) particle pairs for the large particle of type A as a function of the density of small particles (the density of small particles correspond to the area fraction in Q2D and the volume fraction calculated according to Table 2): (a), (e) correspond to the system AC $\left(\sigma_{l}=16\right.$, $\left.\sigma_{s}=13\right) ;(\mathrm{b})$, (f) correspond to the system $\operatorname{AD}\left(\sigma_{l}=16\right.$, $\left.\sigma_{s}=11\right) ;(\mathrm{c}),(\mathrm{g})$ correspond to the system $\operatorname{AE}\left(\sigma_{l}=16\right.$, $\left.\sigma_{s}=9\right) ;(d)$, (h) correspond to the system $\operatorname{AF}\left(\sigma_{l}=16, \sigma_{s}=7\right)$.

that considering 19 classes or topologies of chains is essential to capture the influence exerted by the geometrical constraints. From our thorough analysis a first conclusion we derive is that the variety of formed aggregates is much higher in Q2D, than in bulky ferrofluids. 
The average chain length has been observed to be small, two-three particles, and practically independent of the small particle area fraction. These observations allow to draw a second conclusion: the "poisoning" effect is less pronounced and exerts basically no influence on the microstructure in Q2D systems, albeit the poisoning effect is crucial for bulky systems, where a noticeable decrease in the chain length can be associated to such effect.

The third conclusion we can draw is that, in the presence of geometrical constraints, our results show that the entropy plays the leading part in the aggregation scenario. One should note that in the calculation of the excluded area interactions, the inter-cluster interactions are not considered. However, in the range of area fractions considered in this work, the good agreement we have found shows that there is no need to resort to more complex descriptions [29-31]. On the other hand, for higher area fractions and dipoledipole interaction parameters, inter-cluster (both sterical and dipole) interactions can be very important, and their adequate theoretical description might be essential to capture the microstructure of magnetic nanocolloids in thin films. The influence of entropy in Q2D systems was found to be so strong that, albeit being more energetically advantageous, the formation of large-large pairs of particles becomes less probable than the formation of large-small pairs when the area fraction of small particles is increased. This implies that by increasing the area fraction of small particles, the small particles take a more active role in the aggregation process. In difference to bulk bidisperse systems, in Q2D systems is quite usual to observe small particles enclosed between two large particles forming part of a chain.

We have shown that for particles with constrained degrees of freedom, striking changes in the microstructure are observed: rings and chains with a significant amount of variously positioned small particles can be observed. This means that for real ferrofluids in monolayers, which have an inherent polydispersity, the formation of even more versatile structures should be expected, and as a consequence, magnetic, rheological and optical properties of such systems in thin layers should be expected to drastically differ from the properties usually observed in bulky ferrofluids.

\section{ACKNOWLEDGMENTS}

This work was carried out with the financial support of FP7-IDEAS-ERC grant PATCHY-COLLOIDS (S. S. K.), grant no. MK 6415.2012.2 from the President of the Russian Federation, Russian Foundation for Basic Research (project nos. 12-02-31334 and 12-02-33106), the Ministry of Education and Science of the Russian Federation (grant no. 2.609.2011), and Ural Federal University development program for the financial support of young scientists (A. B. M.).
APPENDIX

In this section we provide the results of the free energy density functional (6) minimization for all structures. Henceforth, the following convention is used: the number of small particles in the cluster is $m$ and of large ones is $n$; the number of various entropically distinguishable chains is $K(i, n, m)$, the numbers of small-small, large-small and large-large bonds in a chain are denoted by $a, b$, and $c$ respectively; an aggregate area has the functional form of $s(i, n, m)$ for each topological structure $i$ (see Fig. 3). We also provide the area fraction for each class of aggregates as a function of small-large $p_{1}$ and large-large $p_{2}$ particle pair formation probabilities together with the contributions of these clusters into the mass-balance series (14) and (15). We remind that the bond energy vector is $\mathbf{E}=$ $\left(e_{11}, e_{12}, e_{22}\right)$, where $e_{i, j}=\ln \left(q_{0}(i j)\right)$.

$\boldsymbol{i}=\mathbf{1}$. Only large particles in the chains:

$m=0, \quad n=1, \ldots, \infty, \quad K(1, n, m)=1, \quad a_{1}=0$,

$$
\begin{gathered}
b_{1}=0, \quad c_{1}=n-1, \quad s(1, n, m)=s_{2}, \\
p_{1}=\exp \left(v_{1}+e_{12}\right), \quad p_{2}=\exp \left(v_{2}+e_{22}\right), \\
g(1, n, m)=\frac{\exp \left(-e_{22}\right)}{s_{2}} p_{2}^{n}, \\
\sum_{n=1}^{\infty} K(1, n, m) g(1, n, m) m=0, \\
\sum_{n=1}^{\infty} K(1, n, m) g(1, n, m) n=\frac{\exp \left(-e_{22}\right)}{s_{2}} \frac{p_{2}}{\left(1-p_{2}\right)^{2}} .
\end{gathered}
$$

$\boldsymbol{i}=2$. The chains consist of large particles and a small one at the end:

$$
\begin{aligned}
& m=1, \quad n=1, \ldots, \infty, \quad K(2, n, m)=1, \\
& a_{2}=0, \quad b_{2}=1, \quad c_{2}=n-1, \quad s(2, n, m)=s_{2} \text {, } \\
& g(2, n, m)=\frac{\exp \left(-e_{22}\right)}{s_{2}} p_{1} p_{2}^{n}, \\
& \sum_{n=1}^{\infty} K(2, n, m) g(2, n, m) m=\frac{\exp \left(-e_{22}\right)}{s_{2}} \frac{p_{1} p_{2}}{1-p_{2}}, \\
& \sum_{n=1}^{\infty} K(2, n, m) g(2, n, m) n=\frac{\exp \left(-e_{22}\right)}{s_{2}} \frac{p_{1} p_{2}}{\left(1-p_{2}\right)^{2}} \text {. } \\
& \boldsymbol{i}=3 \text {. The chain aggregates are composed of large } \\
& \text { particles and two small ones at the ends: } \\
& m=2, \quad n=1, \ldots, \infty, \quad K(3, n, m)=1, \\
& a_{3}=0, \quad b_{3}=2, \quad c_{3}=n-1, \quad s(3, n, m)=s_{2}, \\
& g(3, n, m)=\frac{\exp \left(-e_{22}\right)}{s_{2}} p_{1}^{2} p_{2}^{n}, \\
& \sum_{n=1}^{\infty} K(3, n, m) g(3, n, m) m=\frac{\exp \left(-e_{22}\right)}{s_{2}} \frac{2 p_{1}^{2} p_{2}}{1-p_{2}}
\end{aligned}
$$




$$
\sum_{n=1}^{\infty} K(3, n, m) g(3, n, m) n=\frac{\exp \left(-e_{22}\right)}{s_{2}} \frac{p_{1}^{2} p_{2}}{\left(1-p_{2}\right)^{2}} \text {. }
$$

$\boldsymbol{i}=\mathbf{4}$. The chain structures consist of large particles and a small one enclosed between two large ones:

$$
\begin{gathered}
m=1, \quad n=2, \ldots, \infty, \quad a_{4}=0, \quad b_{4}=2, \\
c_{4}=n-2, \quad s(4, n, m)=s_{2}^{2} / s_{12}, \\
K(4, n, m)= \begin{cases}\frac{n}{2}, \quad n=2 j, \\
\frac{n-1}{2}, \quad n=2 j+1,\end{cases} \\
g(4, n, m)=\frac{s_{12}}{s_{2}^{2}} \exp \left(e_{12}-2 e_{22}\right) p_{1} p_{2}^{n}, \\
\sum_{n=1}^{\infty} K(4, n, m) g(4, n, m) m \\
=\frac{s_{12}}{s_{2}^{2}} \exp \left(e_{12}-2 e_{22}\right) \frac{p_{1} p_{2}^{2}}{\left(1-p_{2}\right)^{2}\left(1+p_{2}\right)}, \\
\sum_{n=1}^{\infty} K(4, n, m) g(4, n, m) n \\
=\frac{s_{12}}{s_{2}^{2}} \exp \left(e_{12}-2 e_{22}\right) \frac{p_{1} p_{2}^{2}\left(p_{2}^{2}+p_{2}+2\right)}{\left(1-p_{2}\right)^{3}\left(1+p_{2}\right)^{2}} .
\end{gathered}
$$

$\boldsymbol{i}=\mathbf{5}$. The chain aggregates are composed of large particles and two small particles, one of which is enclosed between two large ones, and other small particle is at the end of chain:

$$
\begin{gathered}
m=2, \quad n=2, \ldots, \infty, \quad K(5, n, m)=n-1, \\
a_{5}=0, \quad b_{5}=3, \quad c_{5}=n-2, \quad s(5, n, m)=s_{2}^{2} / s_{12}, \\
g(5, n, m)=\frac{s_{12}}{s_{2}^{2}} \exp \left(e_{12}-2 e_{22}\right) p_{1}^{2} p_{2}^{n}, \\
\quad \sum_{n=1}^{\infty} K(5, n, m) g(5, n, m) m \\
=2 \frac{s_{12}}{s_{2}^{2}} \exp \left(e_{12}-2 e_{22}\right) \frac{p_{1}^{2} p_{2}^{2}}{\left(1-p_{2}\right)^{2}} \\
\sum_{n=1}^{\infty} K(5, n, m) g(5, n, m) n \\
=2 \frac{s_{12}}{s_{2}^{2}} \exp \left(e_{12}-2 e_{22}\right) \frac{p_{1}^{2} p_{2}^{2}}{\left(1-p_{2}\right)^{3}} .
\end{gathered}
$$

$\boldsymbol{i}=\mathbf{6}$. The chain structures consist of large particles and two small ones, which are enclosed between large particles separately:

$$
m=2, \quad n=3, \ldots, \infty, \quad a_{6}=0, \quad b_{6}=4,
$$

$$
\begin{gathered}
c_{6}=n-3, \quad s(6, n, m)=s_{2}^{3} / s_{12}^{2}, \\
K(6, n, m)= \begin{cases}\frac{n(n-2)}{4}, & n=2 j, \\
\frac{(n-1)^{2}}{4}, & n=2 j+1,\end{cases} \\
g(6, n, m)=\frac{s_{12}^{2}}{s_{2}^{3}} \exp \left(2 e_{12}-3 e_{22}\right) p_{1}^{2} p_{2}^{n}, \\
\sum_{n=1}^{\infty} K(6, n, m) g(6, n, m) m \\
=\frac{s_{12}^{2}}{s_{2}^{3}} \exp \left(2 e_{12}-3 e_{22}\right) \frac{p_{1}^{2} p_{2}^{3}}{\left(1-p_{2}\right)^{3}\left(1+p_{2}\right)}, \\
\sum_{n=1}^{\infty} K(6, n, m) g(6, n, m) n \\
=\frac{s_{12}^{2}}{s_{2}^{3}} \exp \left(2 e_{12}-3 e_{22}\right) \frac{p_{1}^{2} p_{2}^{3}\left(p_{2}^{2}+2 p_{2}+3\right)}{\left(1-p_{2}\right)^{4}\left(1+p_{2}\right)^{2}} .
\end{gathered}
$$

$\boldsymbol{i}=7$. Only small particles compose the chain aggregates:

$$
\begin{gathered}
m=1, \ldots, \infty, \quad n=0, \quad K(7, n, m)=1, \\
a_{7}=m-1, \quad b_{7}=0, \quad c_{7}=0, \\
s(7, n, m)=s_{12}^{m} / s_{1}^{m-1}, \\
g(7, n, m)=\frac{\exp \left(-e_{11}\right)}{s_{1}}\left(\frac{s_{1}}{s_{12}} \exp \left(e_{11}-e_{12}\right) p_{1}\right)^{m}, \\
\sum_{m=1}^{\infty} K(7, n, m) g(n, 0, m) \cdot 0=0, \\
\sum_{m=1}^{\infty} K(7, n, m) g(n, 0, m) m=\frac{\exp \left(-e_{11}\right)}{s_{1}} \\
\times \frac{\left(s_{1} / s_{12}\right) \exp \left(e_{11}-e_{12}\right) p_{1}}{\left[1-\left(s_{1} / s_{12}\right) \exp \left(e_{11}-e_{12}\right) p_{1}\right]^{2}} .
\end{gathered}
$$

$\boldsymbol{i}=\mathbf{8}$. The chains are composed of large particles and two small ones located sequentially at one of the chain ends:

$$
\begin{gathered}
m=2, \quad n=1, \ldots, \infty, \quad K(8, n, m)=1, \quad a_{8}=1, \\
b_{8}=1, \quad c_{8}=n-1, \quad s(8, n, m)=s_{12} s_{2} / s_{1}, \\
g(8, n, m)=\frac{s_{1}}{s_{12} s_{2}} \exp \left(e_{11}-e_{12}-e_{22}\right) p_{1}^{2} p_{2}^{n}, \\
\sum_{n=1}^{\infty} K(8, n, m) g(8, n, m) m \\
=2 \frac{s_{1}}{s_{12} s_{2}} \exp \left(e_{11}-e_{12}-e_{22}\right) \frac{p_{1}^{2} p_{2}}{1-p_{2}},
\end{gathered}
$$




$$
\begin{gathered}
\sum_{n=1}^{\infty} K(8, n, m) g(8, n, m) n \\
=\frac{s_{1}}{s_{12} s_{2}} \exp \left(e_{11}-e_{12}-e_{22}\right) \frac{p_{1}^{2} p_{2}}{\left(1-p_{2}\right)^{2}} .
\end{gathered}
$$

$\boldsymbol{i}=9$. The chain aggregates consist of large particles and three small ones, two of which are located sequentially at one of the chain ends, and third small particle is located at other end of chain:

$$
\begin{gathered}
m=3, \quad n=1, \ldots, \infty, \quad K(9, n, m)=1, \quad a_{9}=1, \\
b_{9}=2, \quad c_{9}=n-1, \quad s(9, n, m)=s_{12} s_{2} / s_{1}, \\
g(9, n, m)=\frac{s_{1}}{s_{12} s_{2}} \exp \left(e_{11}-e_{12}-e_{22}\right) p_{1}^{3} p_{2}^{n}, \\
\quad \sum_{n=1}^{\infty} K(9, n, m) g(9, n, m) m \\
=3 \frac{s_{1}}{s_{12} s_{2}} \exp \left(e_{11}-e_{12}-e_{22}\right) \frac{p_{1}^{3} p_{2}}{1-p_{2}}, \\
\sum_{n=1}^{\infty} K(9, n, m) g(9, n, m) n \\
=\frac{s_{1}}{s_{12} s_{2}} \exp \left(e_{11}-e_{12}-e_{22}\right) \frac{p_{1}^{3} p_{2}}{\left(1-p_{2}\right)^{2}} .
\end{gathered}
$$

$\boldsymbol{i}=\mathbf{1 0}$. The chain structures are composed of large particles and three small ones, two of which are located at the ends of chain separately, and third small particle is enclosed between large ones:

$$
\begin{gathered}
m=3, \quad n=2, \ldots, \infty, \quad a_{10}=0, \quad b_{10}=4, \\
c_{10}=n-2, \quad s(10, n, m)=s_{2}^{2} / s_{12}, \\
K(10, n, m)=\left\{\begin{array}{l}
\frac{n}{2}, \quad n=2 j, \\
\frac{n-1}{2}, \quad n=2 j+1,
\end{array}\right. \\
g(10, n, m)=\frac{s_{12}}{s_{2}^{2}} \exp \left(e_{12}-2 e_{22}\right) p_{1}^{3} p_{2}^{n}, \\
=3 \frac{s_{12}^{2}}{s_{2}^{2}} \exp \left(e_{12}-2 e_{22}\right) \frac{p_{1}^{3} p_{2}^{2}}{\left(1-p_{2}\right)^{2}\left(1+p_{2}\right)} \\
\sum_{n=1}^{\infty} K(10, n, m) g(10, n, m) m \\
\sum_{n=1}^{\infty} K(10, n, m) g(10, n, m) n \\
s_{2}^{2} \exp \left(e_{12}-2 e_{22}\right) \frac{p_{1}^{3} p_{2}^{2}\left(p_{2}^{2}+p_{2}+2\right)}{\left(1-p_{2}\right)^{3}\left(1+p_{2}\right)^{2}} .
\end{gathered}
$$

$\boldsymbol{i}=\mathbf{1 1}$. The chain aggregates consist of large particles and three small ones, two of which are located sequentially at one of the chain ends, and third small particle is enclosed between large ones:

$$
\begin{gathered}
m=3, \quad n=2, \ldots, \infty, \quad K(11, n, m)=n-1, \\
a_{11}=1, \quad b_{11}=3, \quad c_{11}=n-2, \\
s(11, n, m)=s_{2}^{2} / s_{1}, \\
g(11, n, m)=\frac{s_{1}}{s_{2}^{2}} \exp \left(e_{11}-2 e_{22}\right) p_{1}^{3} p_{2}^{n}, \\
\sum_{n=1}^{\infty} K(11, n, m) g(11, n, m) m \\
=3 \frac{s_{1}}{2} \exp \left(e_{11}-2 e_{22}\right) \frac{p_{1}^{3} p_{2}^{2}}{\left(1-p_{2}\right)^{2}} \\
s_{2}^{\infty} \\
\sum_{n=1}^{\infty} K(11, n, m) g(11, n, m) n \\
=2 \frac{s_{1}}{2} \exp \left(e_{11}-2 e_{22}\right) \frac{p_{1}^{3} p_{2}^{2}}{\left(1-p_{2}\right)^{3}} .
\end{gathered}
$$

$\boldsymbol{i}=\mathbf{1 2}$. The chain structures are composed of large particles and two small ones located within chain together:

$$
\begin{gathered}
m=2, \quad n=2, \ldots, \infty, \quad K(12, n, m)=n-1, \\
a_{12}=1, \quad b_{12}=2, \quad c_{12}=n-2, \\
s(12, n, m)=s_{2}^{2} / s_{1}, \\
g(12, n, m)=\frac{s_{1}}{s_{2}} \exp \left(e_{11}-2 e_{22}\right) p_{1}^{2} p_{2}^{n}, \\
\sum_{n=1}^{\infty} K(12, n, m) g(12, n, m) m \\
=2 \frac{s_{1}}{2} \exp \left(e_{11}-2 e_{22}\right) \frac{p_{1}^{2} p_{2}^{2}}{\left(1-p_{2}\right)^{2}} \\
\sum_{2}^{\infty} K(12, n, m) g(12, n, m) \\
=2 \frac{s_{1}}{s_{2}^{2}} \exp \left(e_{11}-2 e_{22}\right) \frac{p_{1}^{2} p_{2}^{2}}{\left(1-p_{2}\right)^{3}} .
\end{gathered}
$$

$\boldsymbol{i}=\mathbf{1 3}$. The chain aggregates consist of large particles and three small ones located sequentially at one of the chain ends:

$$
\begin{gathered}
m=3, \quad n=1, \ldots, \infty, \quad K(13, n, m)=1, \\
a_{13}=2, \quad b_{13}=1, \quad c_{13}=n-1, \\
s(13, n, m)=s_{12}^{2} s_{2} / s_{1}^{2},
\end{gathered}
$$




$$
\begin{gathered}
g(13, n, m)=\frac{s_{1}^{2}}{s_{12}^{2} s_{2}} \exp \left(2 e_{11}-2 e_{12}-e_{22}\right) p_{1}^{3} p_{2}^{n}, \\
\sum_{n=1}^{\infty} K(13, n, m) g(13, n, m) m \\
=3 \frac{s_{1}^{2}}{s_{12}^{2} s_{2}} \exp \left(2 e_{11}-2 e_{12}-e_{22}\right) \frac{p_{1}^{3} p_{2}}{1-p_{2}}, \\
\sum_{n=1}^{\infty} K(13, n, m) g(13, n, m) n \\
=\frac{s_{1}^{2}}{s_{12}^{2} s_{2}} \exp \left(2 e_{11}-2 e_{12}-e_{22}\right) \frac{p_{1}^{3} p_{2}}{\left(1-p_{2}\right)^{2}} .
\end{gathered}
$$

$\boldsymbol{i}=\mathbf{1 4}$. The chains are composed of large particles and three small ones locates within chain together:

$$
\begin{gathered}
m=3, \quad n=2, \ldots, \infty, \quad K(14, n, m)=n-1, \\
a_{14}=2, \quad b_{14}=2, \quad c_{14}=n-2, \\
s(14, n, m)=s_{12} s_{2}^{2} / s_{1}^{2}, \\
g(14, n, m)=\frac{s_{1}^{2}}{s_{12} s_{2}^{2}} \exp \left(2 e_{11}-e_{12}-2 e_{22}\right) p_{1}^{3} p_{2}^{n}, \\
\sum_{n=1}^{\infty} K(14, n, m) g(14, n, m) m \\
=3 \frac{s_{1}^{2}}{s_{12}^{2} s_{2}^{2}} \exp \left(2 e_{11}-e_{12}-2 e_{22}\right) \frac{p_{1}^{3} p_{2}^{2}}{\left(1-p_{2}\right)^{2}}, \\
\sum_{n=1}^{\infty} K(14, n, m) g(14, n, m) n \\
=2 \frac{s_{1}^{2}}{s_{12} s_{2}^{2}} \exp \left(2 e_{11}-e_{12}-2 e_{22}\right) \frac{p_{1}^{3} p_{2}^{2}}{\left(1-p_{2}\right)^{3}} .
\end{gathered}
$$

$\boldsymbol{i}=\mathbf{1 5}$. The chain structures consist of large particles and four small ones, three of which are located sequentially at one of the chain ends, and fourth small particle is enclosed between large ones:

$$
\begin{gathered}
m=4, \quad n=2, \ldots, \infty, \quad K(15, n, m)=n-1, \\
a_{15}=2, \quad b_{15}=3, \quad c_{15}=n-2, \\
s(15, n, m)=\frac{s_{12} s_{2}^{2}}{s_{1}^{2}} \\
g(15, n, m)=\frac{s_{1}^{2}}{s_{12} s_{2}^{2}} \exp \left(2 e_{11}-e_{12}-2 e_{22}\right) p_{1}^{4} p_{2}^{n}, \\
\sum_{n=1}^{\infty} K(15, n, m) g(15, n, m) m
\end{gathered}
$$

$$
\begin{aligned}
& =4 \frac{s_{1}^{2}}{s_{12} s_{2}^{2}} \exp \left(2 e_{11}-e_{12}-2 e_{22}\right) \frac{p_{1}^{4} p_{2}^{2}}{\left(1-p_{2}\right)^{2}}, \\
& \sum_{n=1}^{\infty} K(15, n, m) g(15, n, m) n \\
& =2 \frac{s_{1}^{2}}{s_{12} s_{2}^{2}} \exp \left(2 e_{11}-e_{12}-2 e_{22}\right) \frac{p_{1}^{4} p_{2}^{2}}{\left(1-p_{2}\right)^{3}} .
\end{aligned}
$$

$\boldsymbol{i}=\mathbf{1 6}$. The chains are composed of large particles and three small ones, two of which are located within chain together, and third small particle is located within chain, but separately from first two ones:

$$
\begin{gathered}
m=3, \quad n=3, \ldots, \infty, \\
K(16, n, m)=\frac{(n-1)(n-2)}{2}, \quad a_{16}=1, \\
b_{16}=4, \quad c_{16}=n-3, \quad s(16, n, m)=s_{2}^{3} / s_{1} s_{12}, \\
g(16, n, m)=\frac{s_{1} s_{12}}{s_{2}^{3}} \exp \left(e_{11}+e_{12}-3 e_{22}\right) p_{1}^{3} p_{2}^{n}, \\
\sum_{n=1}^{\infty} K(16, n, m) g(16, n, m) m \\
=3 \frac{s_{1} s_{12}}{s_{2}^{2}} \exp \left(e_{11}+e_{12}-3 e_{22}\right) \frac{p_{1}^{3} p_{2}^{3}}{\left(1-p_{2}\right)^{3}}, \\
\sum_{n=1}^{\infty} K(16, n, m) g(16, n, m) n \\
=3 \frac{s_{1} s_{12}}{s_{2}^{3}} \exp \left(e_{11}+e_{12}-3 e_{22}\right) \frac{p_{1}^{3} p_{2}^{3}}{\left(1-p_{2}\right)^{4}} .
\end{gathered}
$$

$\boldsymbol{i}=\mathbf{1 7}$. The chain aggregates are composed of large particles and three small ones, which are located within chain separately:

$$
\begin{gathered}
m=3, \quad n=4, \ldots, \infty, \quad a_{17}=0, \quad b_{17}=6, \\
c_{17}=n-4, \quad s(17, n, m)=s_{2}^{4} / s_{12}^{3}, \\
K(17, n, m)= \begin{cases}\frac{(n-2)\left(n^{2}-4 n+6\right)}{12}, & n=2 j, \\
\frac{(n-1)(n-2)(n-3)}{12}, & n=2 j+1,\end{cases} \\
g(17, n, m)=\frac{s_{12}^{3}}{s_{2}^{4}} \exp \left(3 e_{12}-4 e_{22}\right) p_{1}^{3} p_{2}^{n}, \\
\sum_{n=1}^{\infty} K(17, n, m) g(17, n, m) m \\
=3 \frac{s_{12}^{3}}{s_{2}^{4}} \exp \left(3 e_{12}-4 e_{22}\right) \frac{p_{1}^{3} p_{2}^{4}\left(p_{2}^{2}+1\right)}{\left(1-p_{2}\right)^{4}\left(1+p_{2}\right)^{2}},
\end{gathered}
$$




$$
\begin{gathered}
\sum_{n=1}^{\infty} K(17, n, m) g(17, n, m) n \\
=2 \frac{s_{12}^{3}}{s_{2}^{4}} \exp \left(3 e_{12}-4 e_{22}\right) \frac{p_{1}^{3} p_{2}^{4}\left(p_{2}^{3}+4 p_{2}^{2}+p_{2}+2\right)}{\left(1-p_{2}\right)^{5}\left(1+p_{2}\right)^{3}} .
\end{gathered}
$$

$\boldsymbol{i}=18$. The chains consist of large particles and three small ones, two of which are located within chain together, and third small one is located at one of the chain ends:

$$
\begin{gathered}
m=3, \quad n=2, \ldots, \infty, \quad K(18, n, m)=n-1, \\
a_{18}=1, \quad b_{18}=3, \quad c_{18}=n-2, \\
s(18, n, m)=s_{2}^{2} / s_{1}, \\
g(18, n, m)=\frac{s_{1}}{s_{2}} \exp \left(e_{11}-2 e_{22}\right) p_{1}^{3} p_{2}^{n}, \\
\sum_{n=1}^{\infty} K(18, n, m) g(18, n, m) m \\
=3 \frac{s_{1}}{2} \exp \left(e_{11}-2 e_{22}\right) \frac{p_{1}^{3} p_{2}^{2}}{\left(1-p_{2}\right)^{2}} \\
\sum_{2}^{\infty} K(18, n, m) g(18, n, m) n \\
=2 \frac{s_{1}}{s_{2}^{2}} \exp \left(e_{11}-2 e_{22}\right) \frac{p_{1}^{3} p_{2}^{2}}{\left(1-p_{2}\right)^{3}} .
\end{gathered}
$$

$\boldsymbol{i}=\mathbf{1 9}$. The chain structures are composed of large particles and three small ones, two of which are located within chain separately, and third small one is located at the end of chain:

$$
\begin{gathered}
m=3, \quad n=3, \ldots, \infty \\
K(19, n, m)=\frac{(n-1)(n-2)}{2}, \quad a_{19}=0 \\
b_{19}=5, \quad c_{19}=n-3, \quad s(19, n, m)=s_{2}^{3} / s_{12}^{2}, \\
g(19, n, m)=\frac{s_{12}^{2}}{s_{2}^{3}} \exp \left(2 e_{12}-3 e_{22}\right) p_{1}^{3} p_{2}^{n}, \\
\sum_{n=1}^{\infty} K(19, n, m) g(19, n, m) m \\
=3 \frac{s_{12}^{2}}{s_{2}^{3}} \exp \left(2 e_{12}-3 e_{22}\right) \frac{p_{1}^{3} p_{2}^{3}}{\left(1-p_{2}\right)^{3}} \\
\sum_{n=1}^{\infty} K(19, n, m) g(19, n, m) n \\
=3 \frac{s_{12}^{2}}{s_{2}^{3}} \exp \left(2 e_{12}-3 e_{22}\right) \frac{p_{1}^{3} p_{2}^{3}}{\left(1-p_{2}\right)^{4}} .
\end{gathered}
$$

And, finally, for rings we write:

$$
\begin{gathered}
m=0, \quad n=5, \ldots, \infty \\
\sum_{n=5}^{\infty} f(n) n=\sum_{n=5}^{\infty} \frac{\exp \left(n \vee_{2}\right) w_{0}^{n}}{s(n)}
\end{gathered}
$$

\section{REFERENCES}

1. R. Rosensweig, Ferrohydrodynamics (Cambridge University Press, Cambridge, 1985).

2. Ferrofluids: Magnetically Controllable Fluids and Their Applications, Ed. by S. Odenbach (Springer-Verlag, New York, 2003).

3. Ch. Alexiou, R. Jurgons, R. Schmid, C. Bergemann, J. Henke, W. Erhardt, E. Huenges, and F. Parak, J. Drug Target 11, 138 (2003).

4. I. Hilger, W. Andra, R. Hergt, R. Hiergeist, and W. A. Kaiser, in Inorganic Materials Recent Advances, Ed. by D. Bahadur, S. Vitta, and O. Prakash (Narosa, New Delhi, 2004).

5. C. Scherer and A. M. Figueiredo, Braz. J. Phys. 35, 718 (2005).

6. M. Klokkenburg, R. P. A. Dullens, W. K. Kegel, B. H. Erné, and A. P. Philipse, Phys. Rev. Lett. 96, 037203 (2006).

7. S. Kantorovich, J. J. Cerdà, and C. Holm, Phys. Chem. Chem. Phys. 10, 1883 (2008).

8. J. Jordanovic and S. Klapp, Phys. Rev. E: Stat., Nonlinear, Soft Matter Phys. 79, 021405 (2009).

9. P. J. Camp and G. N. Patey, Phys. Rev. E: Stat. Phys., Plasmas, Fluids, Relat. Interdiscip. Top. 62, 5403 (2000).

10. A. Yu. Zubarev and L. Yu. Iskakova, JETP 80 (5), 857 (1995).

11. A. YU. Zubarev, JETP 93 (1), 80 (2001).

12. A. Ivanov and S. Kantorovich, Phys. Rev. E: Stat., Nonlinear, Soft Matter Phys. 70, 021401 (2004).

13. C. Holm, A. Ivanov, S. Kantorovich, and E. Pyanzina, J. Phys.: Condens. Matter 18, 2737 (2006).

14. L. Luo and S. Klapp, J. Chem. Phys. 131, 034709 (2009).

15. T. A. Prokopieva, V. A. Danilov, S. S. Kantorovich, and C. Holm, Phys. Rev. E: Stat., Nonlinear, Soft Matter Phys. 80, 031404 (2009).

16. T. A. Prokop'eva, V. A. Danilov, and S. S. Kantorovich, JETP 113 (3), 435 (2011).

17. A. O. Ivanov, S. S. Kantorovich, E. N. Reznikov, C. Holm, A. F. Pshenichnikov, A. V. Lebedev, A. Chremos, and P. J. Camp, Phys. Rev. E: Stat., Nonlinear, Soft Matter Phys. 75, 061405 (2007).

18. M. P. Allen and D. J. Tildesley, Computer Simulation of Liquids (Oxford University Press, Oxford, 1987).

19. N. Metropolis, A. W. Rosenbluth, M. N. Rosenbluth, A. H. Teller, and E. Teller, J. Chem. Phys. 21, 1087 (1953). 
20. J. D. Weeks, D. Chandler, and H. C. Andersen, J. Chem. Phys. 54, 5237 (1971).

21. J. J. Cerdà, V. Ballenegger, O. Lenz, and C. Holm, J. Chem. Phys. 129, 234104 (2008).

22. A. Arnold, J. de Joannis, and C. J. Holm, J. Chem. Phys. 117, 2496 (2002).

23. P. Ewald, Ann. Phys. (Weinheim) 389, 253 (1921).

24. Z. Wang and C. Holm, J. Chem. Phys. 115, 6351 (2001).

25. H. J. Limbach, A. Arnold, B. A. Mann, and C. Holm, Comput. Phys. Commun. 174, 704 (2006).
26. T. Tlusty and S. A. Safran, Science (Washington) 290, 1328 (2000).

27. S. A. Safran, Nat. Mater. 2, 71 (2003).

28. D. Frenkel and B. Smit, Understanding Molecular Simulation: From Algorithms to Applications (Academic, San Diego, California, United States, 1996).

29. J. Parsons, Phys. Rev. A: At., Mol., Opt. Phys. 19, 1225 (1979).

30. S. J. Lee, J. Chem. Phys. 87, 4972 (1987).

31. C. De Michele, T. Bellini, and F. Sciortino, Macromolecules 45, 1090 (2011). 